\title{
1 Enhanced Solid Tumor Recognition and T cell Stemness with SynNotch CAR Circuits
}

3 Axel Hyrenius-Wittsten ${ }^{1,2}$, Yang Su${ }^{3}$, Minhee Park ${ }^{1}$, Julie M. Garcia ${ }^{1}$, Nathaniel Perry ${ }^{1}$, Garrett

4 Montgomery ${ }^{1}$, Bin Liu ${ }^{3,4^{*}}$, Kole T. Roybal ${ }^{1,2,4,5^{*}}$

$6{ }^{1}$ Department of Microbiology and Immunology, University of California, San Francisco, San

7 Francisco, California, USA

$8 \quad{ }^{2}$ Parker Institute for Cancer Immunotherapy, San Francisco, California, USA

$9{ }^{3}$ Department of Anesthesia, San Francisco, California, USA

$10{ }^{4}$ Helen Diller Family Comprehensive Cancer Center, University of California, San Francisco, San

11 Francisco, California, USA

$12 \quad{ }^{5}$ Chan Zuckerberg Biohub, San Francisco, California, USA

13

14 *Correspondence: kole.roybal@ucsf.edu (KT Roybal), bin.liu@ucsf.edu (B Liu)

16 SHORT TITLE: SynNotch CAR Circuits Enhance T cell Therapy for Solid Tumors 


\section{ABSTRACT}

18 The lack of highly tumor-specific antigens limits the development of engineered T cell therapeutics

19 because of life-threatening "on-target/off-tumor" toxicities. Here we identify ALPPL2 as a tumor-

20 specific antigen expressed in a spectrum of solid tumors, including mesothelioma. ALPPL2 can

21 act as a sole target for chimeric antigen receptor (CAR) therapy or be combined with tumor-

22 associated antigens such as MCAM or mesothelin in synthetic Notch (synNotch) CAR

23 combinatorial antigen circuits. SynNotch CAR T cells display superior tumor control when

24 compared to CAR T cells to the same antigens by prevention of CAR-mediated tonic signaling

25 allowing $\mathrm{T}$ cells to maintain a long-lived memory and non-exhausted phenotype. Collectively, we

26 establish ALPPL2 as a clinically viable target for multiple solid tumors and demonstrate the multi-

27 faceted therapeutic benefits of synNotch CAR T cells.

29 ONE SENTENCE SUMMARY: SynNotch CAR circuits targeting novel solid tumor antigens

30 enhance specificity and improve therapeutic efficacy by regulating $\mathrm{T}$ cell exhaustion. 


\section{MAIN TEXT}

Genetically modified T cells armed with chimeric antigen receptors (CARs) have shown

33 unparalleled clinical efficacy in certain B cell malignancies by targeting lineage-restricted surface

34 molecules (1). Translation of this success to solid tumors has hit roadblocks due to a lack of

35 reliable tumor-specific antigens and sub-optimal therapeutic efficacy. One such treatment refractory solid tumor is mesothelioma - a highly aggressive chronic inflammation-driven cancer with an exceedingly poor prognosis (2). Mesothelioma is inherently hard to treat with traditional modes of cancer therapy and recent trials exploring mono- or combination immune checkpoint

39 inhibitors $(\mathrm{ICl})$ have also had limited impact $(3,4)$. CAR T cells targeting tumor-associated 40 antigens such as mesothelin or fibroblast activation protein have shown preclinical promise, and 41 are now in clinical trials alone or in combination with ICI (NCT02414269 and NCT01722149) (5,

42 6). However, we lack highly tumor-specific antigens with broad coverage of the three major 43 mesothelioma subtypes: epithelioid ( $69 \%$ of cases, median overall survival 14 months), biphasic 44 (12\%, 10 months), and sarcomatoid (19\%, 4 months) (7).

We have now identified Alkaline Phosphatase Placental-like 2 (ALPPL2) as a highly

46 specific and targetable cell surface antigen in all subtypes of mesothelioma (8, 9). ALPPL2 is not

47 only relevant to mesothelioma but is also expressed on other solid tumor types.

48 Immunohistochemistry (IHC) on tumor tissue arrays revealed that ALPPL2 is expressed in $43 \%$

49 of mesothelioma (39/91), $60 \%$ of ovarian cancer (36/60), $36 \%$ of pancreatic cancer (18/50), $18 \%$

50 of gastric cancer (13 of 72) and 100\% of seminoma (11/11) (Fig. 1A and Table S1). Analysis of

$51 \quad \mathrm{HC}$ data from the public protein expression database, the Human Protein Atlas, yielded similar 52 results (Table S1).

53 To design a CAR targeting ALPPL2, we utilized an ALPPL2 binding single-chain variable

54 fragment M25 (8, 9). Second generation anti-ALPPL2 M25 scFv 4-1BB-based (BB $\zeta)$ CARs or two 55 affinity matured clones (M25 $5^{\mathrm{ADLF}}$ and $\left.\mathrm{M} 25^{\mathrm{FYIA}}\right)(9)$ all effectively triggered activation of $\mathrm{CD} 8^{+} \mathrm{T}$ cells 
when stimulated with ALPPL2 positive tumor cells. With the exception of M25 ${ }^{\text {ADLF }}$, CARs carrying M25 or M25 $5^{\mathrm{FYIA}}$ responded with high specificity to ALPPL2 and not the closely related homolog (89\%) ALPI expressed in the healthy intestine (Fig. S1A). To further verify the tissue specificity of $\mathrm{M} 25^{\mathrm{FYIA}}$, an FDA-approved human tissue array that covers 20 different organs in duplicates (40 tissue cores total) was stained with $\mathrm{M} 25^{\mathrm{FYIA}}$ or a control non-binding scFv (YSC10). Positive staining by M25 $5^{\mathrm{FYIA}}$ was only observed in placental trophoblasts, with no staining observed in any other normal tissues (Fig. S1B and Table S2), in line with what we have previously observed for

63 the corresponding full IgG1 antibodies (9). Although the functional role of ALPPL2 in tumor cells

64 is unknown, ALPPL2 expression was recently described to be associated with and functionally

65 essential for the establishment and maintenance of naïve pluripotency in various types of human

66 pluripotent stem cells (10). The broad tumor expression and highly restricted normal tissue

67 expression of ALPPL2, combined with the proposed function of ALPPL2 in various types of stem cells indicates that it might serve as an oncofetal antigen and carry high potential as immunotherapeutic target.

Advances in synthetic biology and immune cell engineering have led to approaches to

71 engineer combinatorial antigen sensing capabilities into therapeutic $\mathrm{T}$ cells (11). We have

72 previously engineered a new class of synthetic receptors based on the Notch receptor we call

73 synNotch that enables custom gene regulation in response to a tissue or disease-related antigenic

74 cue. SynNotch receptors can be engineered to sense a tumor antigen and induce the expression

75 of a CAR to a second tumor-related antigen (12). These synNotch $\rightarrow$ CAR inducible circuits

76 confine CAR expression and thus $\mathrm{T}$ cell activation to the site of disease. Here we sought to

77 develop a clinically relevant synNotch $\rightarrow$ CAR circuit for mesothelioma that senses the

78 combination of the novel tumor-specific antigen ALPPL2 and melanoma cell adhesion molecule

79 (MCAM, also known as CD146 or MUC18) a mesothelioma-associated antigen found expressed

80 in both epithelioid and sarcomatoid mesothelioma, as well as tumor associated blood vessels (8,

81 13). MCAM is reported to be restricted to few normal tissues $(14,15)$. To determine the co- 
82 expression pattern of ALPPL2 and MCAM, we performed dual-antibody IHC on human

83 mesothelioma arrays. Using two different MCAM antibodies (Table S3), we observed $\sim 52-81 \%$

84 MCAM co-staining in ALPPL2 positive tissue cores (Fig. 1B and Table S4). ALPPL2/MCAM cell

85 surface co-expression was also seen on the mesothelioma cell lines M28 (epithelioid) and VAMT-

861 (sarcomatoid) using our anti-ALPPL2 scFv (M25 $\left.5^{\text {FYIA }}\right)$ and anti-MCAM scFv (M1) (Fig. S1C) (16).

87 Indeed, ALPPL2 CAR expressing $\mathrm{CD}^{+} \mathrm{T}$ cells effectively recognized and killed both

88 mesothelioma cell lines (Fig. S1D). Together, these results suggest that the majority of ALPPL2

89 expressing tumors also express MCAM, providing a rationale for dual targeting of ALPPL2/MCAM

90 using an ALPPL2-synNotch design (Fig. 1C). Additionally, for patients with epithelioid

91 mesothelioma, an alternative opportunity would be to use the well-established mesothelioma

92 biomarker mesothelin as a secondary target, thereby reducing the risk of "on-target/off-tumor"

93 toxicity associated with mesothelin (17). This approach would be mainly restricted, but widely

94 applicable, to epithelioid mesothelioma for which mesothelin is found expressed in $84-93 \%$ of

95 patients $(18,19)$ (Fig. 1C).

We first constructed $\mathrm{BB} \zeta \mathrm{CARs}$ targeting either MCAM or mesothelin as well as a CD28

97 based $(28 \zeta)$ mesothelin CAR, which is currently in clinical trials (Fig. S2A and S2B). CD8 ${ }^{+} \mathrm{T}_{\text {cells }}$

98 engineered with the novel CARs and reference CD19 and mesothelin CARs were challenged with

99 tumor cells expressing the corresponding antigens revealing specific activation, robust $\mathrm{T}$ cell

100 proliferation, and production of $\mathrm{Th}_{1}$ immunostimulatory cytokines IL-2, IFN- $\gamma$, and TNF $\alpha$ (Fig. S2C,

101 S2D, S2E, S2F, and S2G). To confine CAR expression locally to mesothelioma tumor tissues, we

102 generated an ALPPL2-sensing synNotch using the M25 $5^{\mathrm{FYIA}}$ scFv and linked it to inducible genetic

103 circuits containing either the MCAM CAR $(\mathrm{BB} \zeta)$ or a mesothelin $\mathrm{CAR}(\mathrm{BB} \zeta$ or $28 \zeta)$ (Fig. 2A). CD8 ${ }^{+}$

104 T cells equipped with these circuits were all able to selectively drive expression of the respective

105 CARs when stimulated with target cells expressing ALPPL2 (Fig. 2B). As with T cells expressing

106 the CARs constitutively, both the ALPPL2 synNotch $\rightarrow$ MCAM CAR and mesothelin CAR circuits 
107 induced robust $\mathrm{T}$ cell activation, proliferation, $\mathrm{Th}_{1}$ cytokine production, and tumor cell killing when 108 combined with target cells expressing the correct ligand combination (Fig. S3A, S3B, S3C, and 109 S3D).

Both constitutive- and ALPPL2-primed expression of MCAM CAR was able to effectively

111 elicit a cytotoxic response towards M28 and VAMT-1 tumor cell lines (Fig. 2C), whereas

112 mesothelin CARs only targeted the epithelioid M28 cell line (Fig. S3E). There was a significant

113 difference in the kinetics of cytotoxicity between constitutive- and circuit-controlled CAR 114 expression, with the latter displaying several fold slower tumor killing rates (Fig. 2D). This more 115 paced cytotoxic response of the synNotch $\rightarrow$ CAR circuit $T$ cells can be attributed to the 116 requirement for transcriptional regulation of CAR expression and as a population the circuit T cells 117 do not upregulate the CAR in perfect unison. Protein levels of ALPPL2 vary within and between 118 tumors based on IHC of various tumor types. Therefore, we wanted to confirm that our ALPPL2119 synNotch remained responsive to lower antigen levels. For this purpose, we designed target cells 120 for which levels of ALPPL2 could be controlled via titration of doxycycline (Fig. 2E and S3F). 121 Using this system, we show that a low level of ALPPL2 is sufficient to induce robust expression 122 of the MCAM CAR through the anti-ALPPL2 M25 $5^{\mathrm{FYIA}}$-based synNotch, allowing for potent T cell 123 activation through combinatorial antigen sensing (Fig. 2E).

124 Another limiting factor in CAR T therapy of solid tumors is the intrinsic maintenance of 125 multifunctional $\mathrm{T}$ cell states and the prevention of $\mathrm{T}$ cell exhaustion/dysfunction. In the absence 126 of antigen, constitutive expression of CARs is known to elicit tonic signaling. This low level 127 signaling is linked to detrimental effects on the phenotypic state of CAR T cells, such as 128 differentiation of long-lived T cell memory phenotypes towards short-lived effector states and the 129 upregulation of inhibitory receptors (20-22). Although most of the components of CAR domain 130 architecture impact tonic signaling (e.g. the scFv, hinge, and signaling domains), surface 131 expression levels remain as the unifying determinant (20). We thus reasoned that restricting the 132 timing of CAR expression and location of expression to the tumor tissue with synNotch could 
133 prevent CAR-mediated tonic signaling and the detrimental effects thereof. We observed that 134 constitutive expression of the MCAM CAR in $\mathrm{CD}^{+} \mathrm{T}$ cells impacted $\mathrm{T}$ cell differentiation and 135 consistently displayed a significantly smaller fraction of long-lived T stem cell memory ( $\left.\mathrm{T}_{\mathrm{SCM}}\right)$ cells 136 (defined as $\mathrm{CCR} 7^{+} \mathrm{CD} 45 \mathrm{RO}^{-} \mathrm{CD} 27^{+} \mathrm{CD} 45 \mathrm{RA}^{+} \mathrm{CD} 95^{+}$) and a higher proportion of T effector memory 137 cells (defined as $\mathrm{CCR}^{-} \mathrm{CD} 45 \mathrm{RO}^{+}$) in contrast to the ALPPL2 synNotch $\rightarrow$ MCAM CAR circuit (Fig. 138 3A and 3B). The same protection from premature $\mathrm{T}$ cell differentiation was observed when 139 comparing ALPPL2-synNotch $\rightarrow$ ALPPL2 CAR or CD19 CAR circuits T cells to T cells that 140 constitutively express the corresponding CARs. Thus, synNotch-mediated CAR regulation is a 141 general means to control tonic signaling and prevent detrimental $\mathrm{T}$ cell differentiation prior to 142 antigen exposure, and this effect remains during long-term ex vivo T cell culture. (Fig. $3 \mathrm{C}$ and 143 S4A). In line with this, constitutive CAR expression led to higher expression and co-expression 144 of surface markers linked to $\mathrm{T}$ cell exhaustion that was avoided by synNotch transcriptional 145 regulation (Fig. 3D, S4B, S4C, and S4D). Upon target cell stimulation, MCAM CAR expression 146 levels were comparable between constitutive and ALPPL2 synNotch transcriptional regulation 147 (Fig. S4E). Further, Jurkat T cell reporter systems for AP-1, NFAT, and NF-кB transcriptional 148 activity showed that constitutive expression of the MCAM CAR was sufficient to induce NF-кB149 mediated transcription (Fig. 3E and S4F). NF-кB transcriptional activity was not observed when 150 the MCAM CAR was under control of the ALPPL2 synNotch suggesting that synNotch regulated 151 CAR expression maintains the engineered $\mathrm{T}$ cell population in a superior functional state by 152 circumventing unfavorable tonic signaling. NF-кB signaling has previously been associated with 153 tonic signaling in $\mathrm{BB} \zeta \mathrm{CARs}$ and has been linked to CAR expression levels (23).

154 To evaluate the therapeutic efficacy of our new CARs and clinically viable synNotch 155 circuits, NSG mice were implanted with epithelioid M28 tumors prior to receiving an infusion of 156 ALPPL2 CAR T cells, MCAM CAR T cells, or ALPPL2 synNotch $\rightarrow$ MCAM-CAR circuit T cells. 157 Constitutive MCAM CAR T cells exhibited inconsistent ability to control M28 tumor growth, 
158 whereas ALPPL2 CAR T showed significant reduction in tumor growth (Fig. 4A). However, the

159 greatest effect was observed for the ALPPL2 synNotch $\rightarrow$ MCAM CAR circuit T cells, for which a 160 complete response was observed in a majority of the mice (Fig. 4A).

161 We then sought to determine the underlying reasons for the enhanced efficacy of the 162 synNotch $\rightarrow$ CAR circuit T cells. We assessed CD4+ and CD8+ engineered T cells in the spleen 163 and determined that the number of T cells correlated with the ability to control tumor growth (Fig. 164 S5A), suggesting that $\mathrm{T}$ cell persistence plays a pivotal role in therapeutic efficacy. $\mathrm{T}$ cell 165 persistence inversely correlated with the level of tonic signaling (fraction CD39+) in the CD4 ${ }^{+}$and $166 \mathrm{CD}^{+}$pre-infusion ALPPL2 synNotch circuit and constitutive CAR T cells (MCAM CAR > ALPPL2 167 CAR $>$ circuit, Fig. S5B). In line with this, ALPPL2 $\rightarrow$ MCAM CAR circuit T cells harvested 9 days 168 post infusion from the spleen were more enriched for long-lived memory phenotypes in both the $169 \mathrm{CD}^{+}$and $\mathrm{CD} 4^{+}$subsets compared constitutive CAR T cells (Fig. 4B and 4C).

170 Here we have explored the multidimensional advantages of synNotch $\rightarrow$ CAR circuits, 171 which circumvent some of the critical problems thought to prevent efficacy of cell therapies for 172 solid tumors. SynNotch $\rightarrow$ CAR circuits not only provide improved specificity through multi-antigen 173 sensing, but are also a general means to enhance therapeutic efficacy through cell-autonomous 174 and context-dependent regulation of CAR expression and prevention of tonic signaling, leading 175 to the maintenance of $\mathrm{T}$ cell memory subsets important for persistence and sustained activity of 176 the cell therapy (Fig. 4D). We also identify ALPPL2 as a highly promising tumor-specific antigen 177 for engineered T cell therapies for a range of solid tumors. ALPPL2 can act as a singular target 178 for CAR T cell therapy or can be sensed in combination with secondary highly expressed tumor179 associated antigens via synNotch $\rightarrow$ CAR circuits to maximize tumor elimination and reduce 180 toxicity potential (Fig. 4E). 
Figure 1.

A

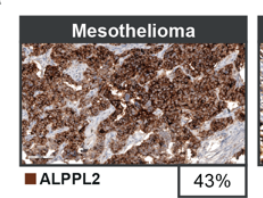

B

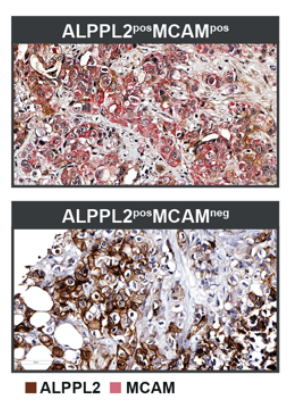

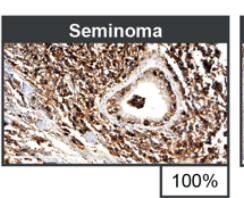

C
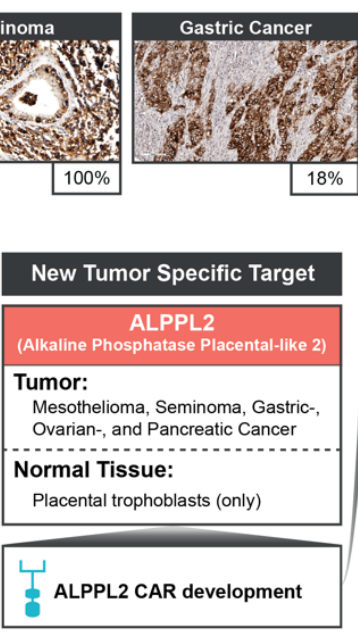
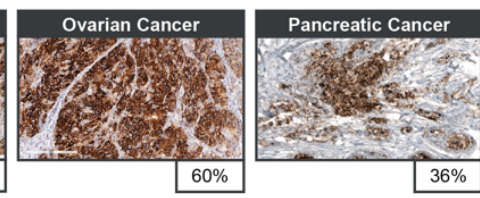

Novel Mesothelioma Antigen Signatures

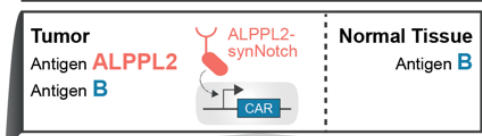

Mesothelioma Subtype Antigen Signatures

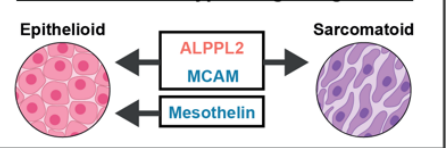

183 Figure 1. ALPPL2 - a highly specific and broadly applicable tumor antigen for combinatorial antigen recognition of solid tumors with SynNotch CAR T cells. (A) IHC study of ALPPL2 expression in FFPE solid tumor tissues. Positive staining was observed in mesothelioma, seminoma, gastric cancer, ovarian cancer, and pancreatic cancer. Scale bar: 100

$187 \mu \mathrm{m}$ for mesothelioma, pancreatic cancer, and gastric cancer; $200 \mu \mathrm{m}$ for ovarian cancer and seminoma. (B) Representative images of ALPPL2 and MCAM co-expression in mesothelioma

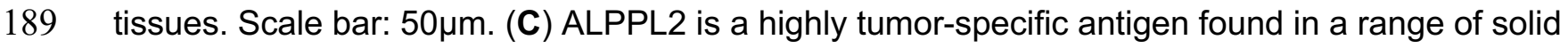

190 tumor types that display minimal expression in normal tissues. ALPPL2 can be targeted directly

191 using an ALPPL2 CAR but can also function as a priming switch for CARs targeting other tumor-

192 associated antigen through synNotch CAR circuits to minimize "on-target/off-tumor" toxicity. We

193 identified ALPPL2/MCAM as an antigen signature with coverage across mesothelioma subtypes.

194 Mesothelin is mainly restricted to epithelioid mesothelioma. 


\section{Figure 2.}

A

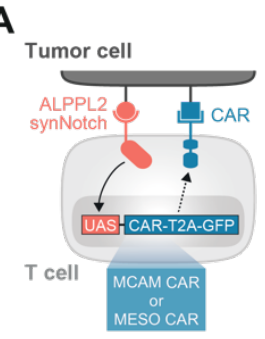

C Epithelioid Mesothelioma (M28)

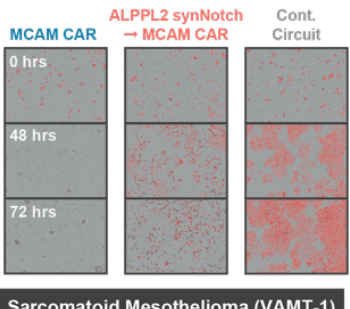

Sarcomatoid Mesothelioma (VAMT-1)

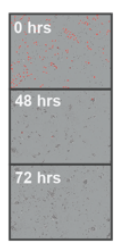

B

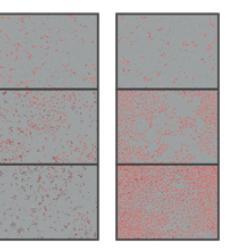

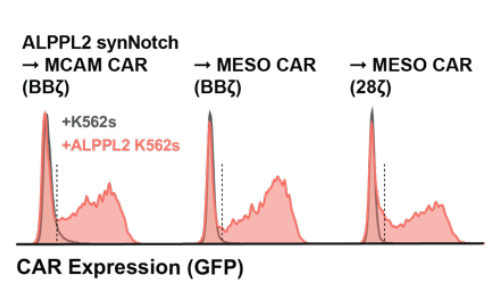

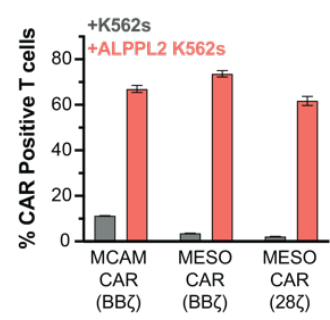

D
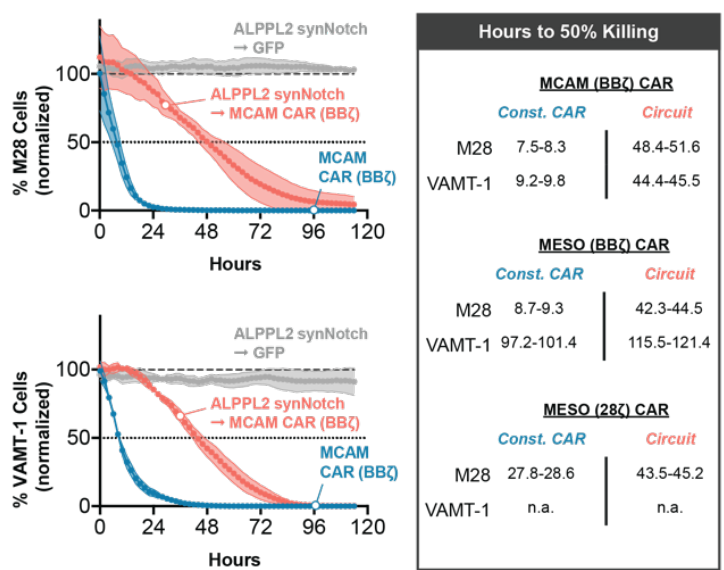

E

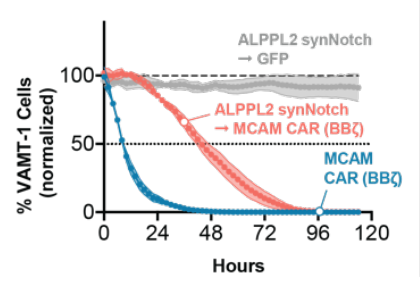

D

\section{A}
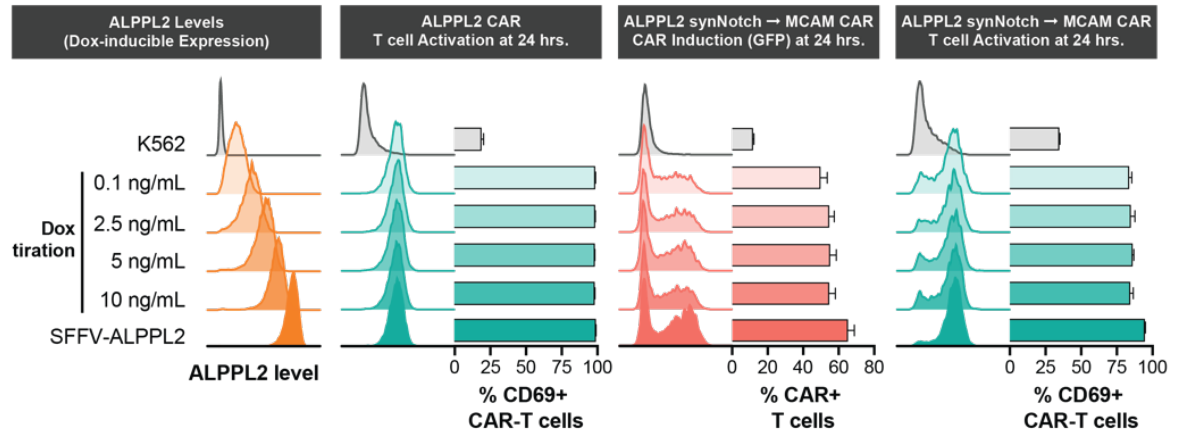

Figure 2. SynNotch CAR circuit T cells exhibit highly sensitive multi-antigen specificity and paced elimination of Mesothelioma. (A) Primary human $\mathrm{CD}^{+} \mathrm{T}$ cells engineered with

198 ALPPL2 sensing synNotch with genetic circuits encoding for CARs targeting either MCAM (BB $\zeta)$

199 or mesothelin (BB $\zeta$ or $28 \zeta$ ) CARs. (B) Antigen specific CAR expression was determined by GFP

200 levels after $24 \mathrm{hrs}$ of stimulation with K562s expressing MCAM and mesothelin +/- ALPPL2 (C)

201 Killing kinetics of epithelioid (M28) and sarcomatoid (VAMT-1) mesothelioma tumor cells by CD8 ${ }^{+}$ 
202 T cells expressing a MCAM CAR constitutively or through an ALPPL2-synNotch circuit. Control

203 circuit (Cont. circuit) is an ALPPL2-synNotch regulating GFP expression. (D) Hours to $50 \%$ killing

204 of M28 and VAMT-1, as compared to untransduced CD8 ${ }^{+}$T cells, for constitutive or ALPPL2

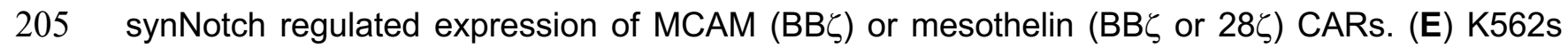

206 constructed with doxycycline-inducible ALPPL2 expression showing dose-dependent induction of

207 ALPPL2 after 72 hrs of doxycycline treatment. CD8 ${ }^{+}$T cells engineered with an ALPPL2 CAR or

208 ALPPL2-synNotch MCAM CAR circuit were challenged with K562s displaying a range of ALPPL2

209 expression levels and analyzed for antigen specific CAR expression, as determined by GFP

210 expression, and T cell activation, as determined by CD69 expression. n.a.; not applicable. Data

211 is shown as mean \pm SD. 
Figure 3.

A

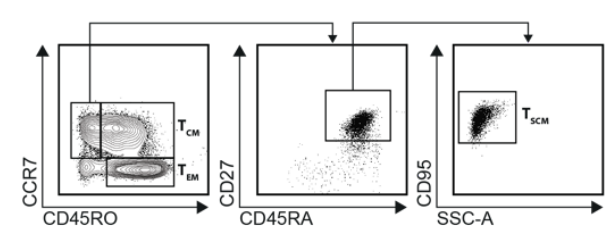

B

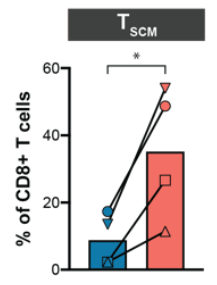

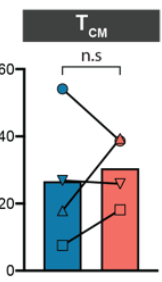

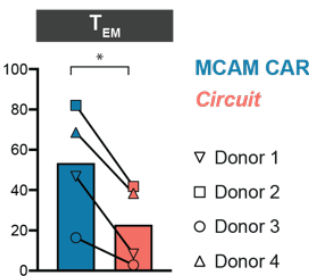

C

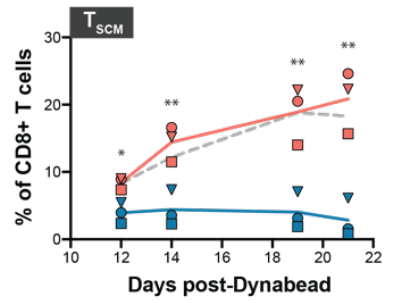

D

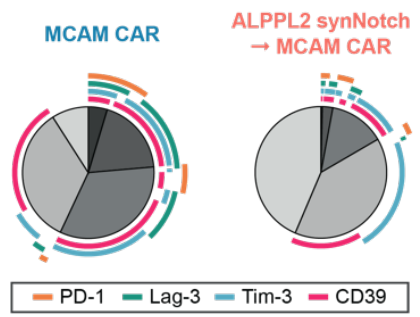

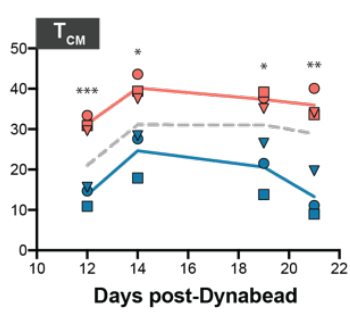

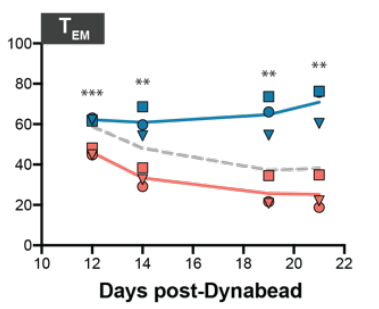

CAR

Circuit

Untr. cells

$\square$ MCAM CAR

$\nabla$ ALPPL2 CAR O CD19 CAR

E
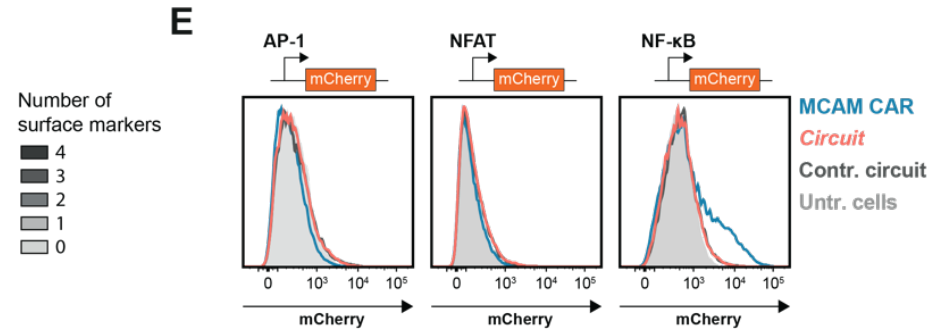

E

213 Figure 3. SynNotch regulation of CAR expression is a general means to maintain $T$ cell

214 stemness prior to therapeutic administration. (A) Gating strategy for identifying T stem cell

215 memory $\left(\mathrm{T}_{\mathrm{SCM}}\right)$, $\mathrm{T}$ central memory $\left(\mathrm{T}_{\mathrm{CM}}\right)$, and $\mathrm{T}$ effector memory $\left(\mathrm{T}_{\mathrm{EM}}\right)$ cells. (B) Composition of

$216 \mathrm{~T}_{\mathrm{SCM}}, \mathrm{T}_{\mathrm{CM}}$, and $\mathrm{T}_{\mathrm{EM}}$ in non-antigen exposed $\mathrm{CD}^{+} \mathrm{T}$ cells from four different donors engineered to

217 express a MCAM CAR either constitutively or through an ALPPL2-synNotch circuit 14 days post

218 initial CD3/CD28 Dynabead stimulation. (C) Longitudinal T cell memory subsetting of donor 4

219 engineered to express either an MCAM, ALPPL2, or CD19 CAR constitutively or through ALPPL2-

220 synNotch circuits. (D) Expressional pattern analysis of CD39, Lag-3, PD-1, Tim-3 in non-antigen

221 exposed $\mathrm{CD}^{+} \mathrm{T}$ cells from three different donors engineered to express a MCAM-CAR either

222 constitutively or through ALPPL2 synNotch circuit 14 days post initial CD3/CD28 Dynabead

223 stimulation. (E) Jurkats carrying AP-1, NFAT, or NF-kB response elements expressing a MCAM 
bioRxiv preprint doi: https://doi.org/10.1101/2021.01.06.425642; this version posted January 7, 2021. The copyright holder for this preprint (which was not certified by peer review) is the author/funder. All rights reserved. No reuse allowed without permission.

224 CAR constitutively or through ALPPL2-synNotch circuit. Control circuit is ALPPL2 synNotch 225 driving GFP expression. Statistics was calculated using paired (B) or unpaired (C) Student's t226 test. ${ }^{*} \mathrm{P} \leq 0.05 ;{ }^{* *} \mathrm{P} \leq 0.01 ;{ }^{* * *} \mathrm{P} \leq 0.001$; n.s.; not significant. 
Figure 4.

A
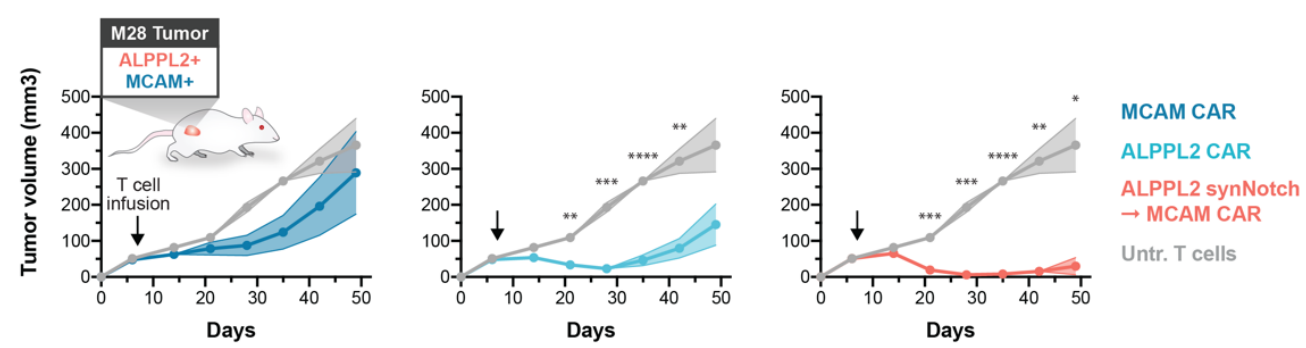

B
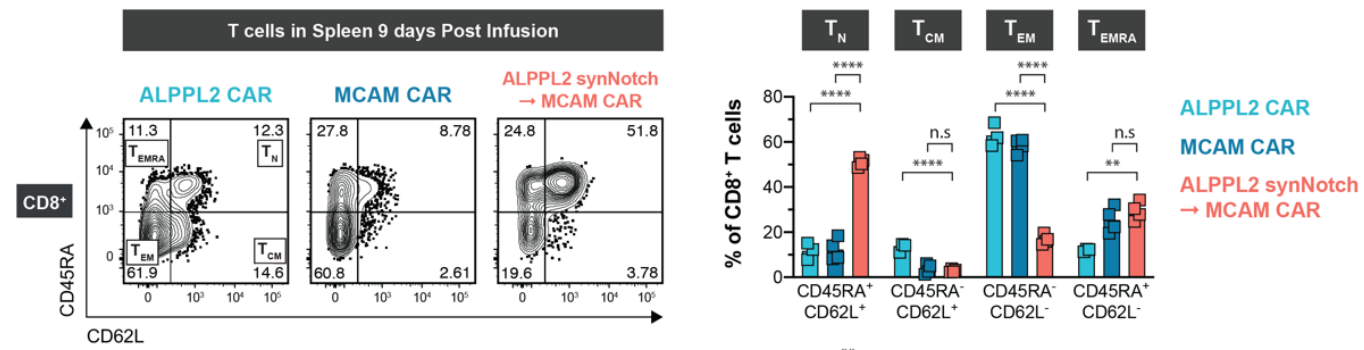

C
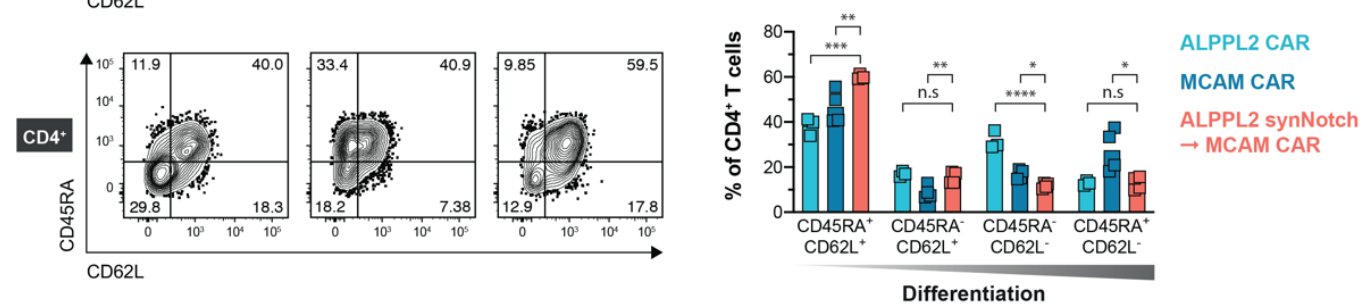

D

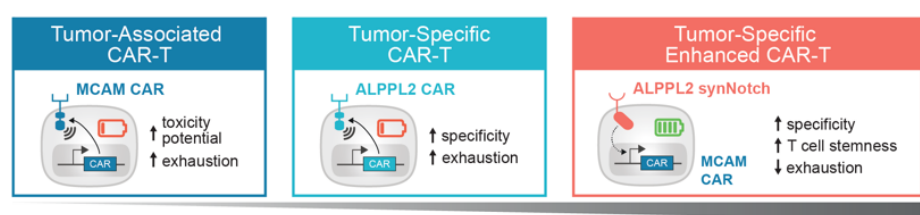

Therapeutic Potential
E

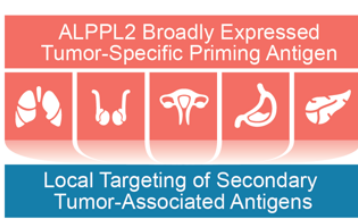

Figure 4. SynNotch CAR Circuit T cells exhibit superior efficacy and persistence in vivo.

229 (A) NSG subcutaneously implanted with M28 tumor cells were injected i.v. with 1:1 ratio of

$230 \mathrm{CD}^{+}: \mathrm{CD}^{+}$T cells engineered with an ALPPL2 CAR $(n=5)$, MCAM CAR ( $\left.n=5\right)$, ALPPL2 synNotch

231 regulating MCAM-CAR expression, or untransduced T cells $(n=5)$. Tumor size was monitored

232 over 49 days. Data is presented as mean \pm SEM. (B,C) Percentage of $\mathrm{T}$ cell memory subsets in

233 (B) $\mathrm{CD}^{+}$and (C) $\mathrm{CD}^{+}$in the spleen 9 days after T cell infusion. (D) SynNotch CAR circuits allow

234 for safe targeting of tumor-associated antigens and enhances immunotherapeutic potential by

235 avoiding tonic CAR signaling outside tumor tissues. (E) ALPPL2 is a novel highly tumor-specific 
236 antigen expressed in mesothelioma, seminoma, gastric-, ovarian-, and pancreatic cancer that

237 allows for safe targeting of other less specific tumor-associated antigens. Statistics were

238 calculated using either two-way ANOVA with Tukey's multiple comparison (A) or one-way ANOVA

239 with Dunnett's multiple comparisons test $(\mathbf{B}, \mathbf{C}) .{ }^{*} \mathrm{P} \leq 0.05 ;{ }^{* *} \mathrm{P} \leq 0.01$; ${ }^{* *} \mathrm{P} \leq 0.001 ;{ }^{* *} \mathrm{P}<0.0001$;

240 n.s.; not significant. 


\section{NOTES}

\section{Acknowledgements}

243 A.H-W. has received funding from the Swedish Society for Medical Research and the Swedish

244 Research Council. K.T.R. is funded by the Parker Institute for Cancer Immunotherapy, the UCSF

245 Helen Diller Family Comprehensive Cancer Center, the Chan Zuckerberg Biohub, an NIH

246 Director's New Innovator Award (DP2 CA239143), Cancer Research UK, and the Kleberg

247 Foundation. We acknowledge the PFCC supported in part by Grant NIH P30 DK063720 and by

248 the NIH S10 Instrumentation Grant S10 1S100D021822-01.

\section{Author Contributions}

251 A.H.W. designed the study, designed and performed experiments and vector construction, 252 analyzed data, and wrote the manuscript; Y.S. performed and analyzed histological stains, 253 assisted in in vivo experiments, and wrote the manuscript; M.P., N.P., and G.M. performed vector 254 construction; M.P. and J.G. performed in vitro experiments; B.L. and K.T.R. conceived and 255 designed the study, designed experiments, and wrote the manuscript.

\section{Competing Interests}

258 K.T.R. is a cofounder of Arsenal Biosciences. K.T.R. is an inventor on patents for synthetic Notch 259 receptors (WO2016138034A1, PRV/2016/62/333,106) and receives licensing fees and royalties.

260 The patents were licensed by Cell Design Labs and are now part of Gilead. He was a founding 261 scientist/consultant and stockholder in Cell Design Labs, now a Gilead Company. K.T.R. holds 262 stock in Gilead. The remaining authors declare no competing financial interests. 
bioRxiv preprint doi: https://doi.org/10.1101/2021.01.06.425642; this version posted January 7, 2021. The copyright holder for this preprint (which was not certified by peer review) is the author/funder. All rights reserved. No reuse allowed without permission.

\section{SUPPLEMENTARY MATERIALS}

Materials and Methods

Tables S1-S4

266

Figs. S1-S4

267

References (1-27) 


\section{References}

269 1. E. Jacoby et al., Immunol. Rev. 290, 39-59 (2019).

270 2. M. Carbone et al., CA Cancer J Clin. 69, 402-429 (2019).

271 3. F. Nicolini et al., Front Oncol. 9, 1519 (2019).

272 4. C. J. de Gooijer et al., Front Oncol. 10, 187 (2020).

273 5. P. S. Adusumilli et al., Sci Transl Med. 6, 261ra151-261ra151 (2014).

$274 \quad$ 6. P. C. Schuberth et al., J Transl Med. 11, 187 (2013).

275 7. R. R. Meyerhoff et al., J. Surg. Res. 196, 23-32 (2015).

276 8. F. An et al., Mol. Cancer Ther. 7, 569-578 (2008).

277 9. Y. Su et al., bioRxiv, 2020.01.07.898122 (2020).

278 10. Y. Bi et al., Cell Rep. 30, 3917-3931.e5 (2020).

279 11. A. Hyrenius-Wittsten et al., Trends Cancer. 5, 583-592 (2019).

280 12. K. T. Roybal et al., Cell. 164, 770-779 (2016).

281 13. S. Bidlingmaier et al., Cancer Res. 69, 1570-1577 (2009).

282 14. I. M. Shih et al., Mod. Pathol. 11, 1098-1106 (1998).

283 15. X. Yan et al., Blood. 102, 184-191 (2003).

284 16. R. A. Metcalf et al., Cancer Res. 52, 2610-2615 (1992).

285 17. J. Lv et al., Biomark Res. 7, 18 (2019).

286 18. T. Eguchi et al., Oncotarget. 8, 77872-77882 (2017).

287 19. S. Inaguma et al., Oncotarget. 8, 26744-26754 (2017).

288 20. A. Ajina et al., Mol. Cancer Ther. 17, 1795-1815 (2018).

289 21. J. Eyquem et al., Nature. 543, 113-117 (2017).

290 22. A. H. Long et al., Nat. Med. 21, 581-590 (2015).

291 23. D. Gomes-Silva et al., Cell Rep. 21, 17-26 (2017). 
298 Axel Hyrenius-Wittsten ${ }^{1,2}$, Yang Su${ }^{3}$, Minhee Park ${ }^{1}$, Julie M. Garcia ${ }^{1}$, Nathaniel Perry ${ }^{1}$, Garrett

299 Montgomery ${ }^{1}$, Bin Liu $^{3,4^{*}}$, Kole T. Roybal ${ }^{1,2,4,5^{*}}$

300 *Correspondence: kole.roybal@ucsf.edu (KT Roybal), bin.liu@ucsf.edu (B Liu) 


\section{MATERIALS AND METHODS}

\section{Recombinant scFv production}

303 ScFv expression and purification was done as previously described $(24,25)$. Briefly, the scFv 304 gene was cloned into the secretion vector pUC119mycHis to impart a c-myc epitope and a 305 hexahistidine tag at the C-terminus. Soluble scFv was harvested from the bacterial periplasmic 306 space and purified by immobilized metal affinity (HiTrap His, GE HealthCare) and ion-exchange

307 (DEAE, GE HealthCare) chromatography.

\section{Immunohistochemistry}

310 IHC studies on frozen tissues were performed as described (9). Briefly, frozen normal human

311 tissue arrays (US Biomax) were air-dried for 15 min at RT, fixed in 4\% paraformaldehyde for 10 312 min, washed 3 times with PBST (PBS containing $0.1 \%$ Tween-20), and incubated with $3 \% \mathrm{H}_{2} \mathrm{O}_{2}$

313 (Thermo Fisher Scientific) for $10 \mathrm{~min}$ to block endogenous peroxidase activity, washed with PBST,

314 further blocked with PBST containing 2\% goat serum (Jackson ImmunoResearch Laboratories)

315 and 5\% BSA (Thermo Fischer Scientific) at RT for $1 \mathrm{~h}$. After avidin/biotin (Vector Laboratories)

316 blocking and washed three times with PBST, slides were incubated with $10 \mu \mathrm{g} / \mathrm{ml}$ biotinylated

317 scFvs (M25 $5^{\mathrm{FYA}}$ and a non-binding $\left.\mathrm{C} 10\right)$ at $4{ }^{\circ} \mathrm{C}$ overnight, followed by detection with streptavidin-

318 HRP (Jackson ImmunoResearch Laboratories) using DAKO liquid DAB+ (Agilent). Slides were

319 counterstained with hematoxylin followed by bluing reagent (Scytek), mounted in Aqua-mount

320 (Lerner Laboratories), and scanned by Aperio AT2 digital scanner (Leica).

$321 \quad$ IHC study on FFPE tissue arrays were performed as described (9). Briefly, FFPE slides

322 were deparaffinized in xylene overnight, rehydrated by sequential exposure to $100 \%, 95 \%, 70 \%$

323 ethanol, and $\mathrm{ddH}_{2} \mathrm{O}$ (7 min each), and placed in Tris Buffer (10 mM Tris Base, $1 \mathrm{mM}$ EDTA, 0.05\%

324 Tween $20, \mathrm{pH} \mathrm{9.0)}$ at $95^{\circ} \mathrm{C}$ for 25 min for antigen retrieval, washed three times with PBST, 325 sequential blocked with $3 \% \mathrm{H}_{2} \mathrm{O}_{2}$ and $2 \%$ donkey serum (Santa Cruz Biotechnology Inc), 326 incubated at $4^{\circ} \mathrm{C}$ overnight with primary antibodies (anti-ALPPL2 mouse mAb (LifeSpan clone 
327 SPM593, catalogue\# LS-C390148-20) at $0.4 \mu \mathrm{g} / \mathrm{ml}$, or anti-MCAM rabbit mAb (Abcam clone 328 EPR3208, catalogue\# ab75769) at $0.625 \mu \mathrm{g} / \mathrm{ml}$, or anti-MCAM rabbit polyclonal antibody (Abcam, 329 catalogue\# ab228487 ) at $10 \mu \mathrm{g} / \mathrm{ml}$ ), washed three times with PBST, further incubated with anti330 mouse DAKO EnVision+ (Agilent) or anti-rabbit DAKO EnVision+ (Agilent), followed detection 331 using DAKO liquid DAB+ (Agilent). Slides were counterstained with hematoxylin followed by 332 bluing reagent (Scytek), dehydrated by sequential exposure to $70 \%, 95 \%, 100 \%$ ethanol, 333 mounted using Permount mounting media (Thermo Fischer Scientific) and scanned by Aperio XT 334 and AT2 digital scanners (Leica).

335 For MCAM/ALPPL2 co-staining on FFPE tissue arrays (US Biomax), the ImmPRESS $\otimes$ 336 Duet Double Staining HRP/AP Polymer Kit was used (HRP anti-Mouse IgG and AP anti-Rabbit 337 IgG, Vector Laboratories). Following deparaffinization and antigen retrieval with Tris Buffer (10 $338 \mathrm{mM}$ Tris Base, $0.05 \%$ Tween 20, $\mathrm{pH}$ 10.0), endogenous peroxidase activity was blocked by 339 BLOXALL blocking solution for 15 min followed by PBST wash. The slides were further blocked 340 with $2.5 \%$ normal horse serum at RT for $1 \mathrm{~h}$, washed three times with PBST and incubated with 341 the following primary antibody pairs diluted into $2.5 \%$ normal horse serum and incubated with the 342 tissue slides at $4^{\circ} \mathrm{C}$ overnight: pair 1: anti-MCAM rabbit mAb and anti-ALPPL2 mouse mAb (both 343 at 1:200 dilution); and pair 2: anti-MCAM rabbit pAb and anti-ALPPL2 mouse mAb (both at 1:100 344 dilution). Slides were washed three times with PBST, incubated with ImmPRESS Duet reagent at 345 RT for 30 min, followed by sequential detection with ImmPACT DAB EqV and ImmPACT Vector 346 Red substrates. Slides were counterstained with hematoxylin followed by bluing reagent (Scytek). 347 After dehydration, the slides were mounted using Permount mounting media (Thermo Fischer 348 Scientific) and scanned by Aperio XT and AT2 digital scanners (Leica).

\section{Vector Construction Designs}

351 All CARs contained CD8 $\alpha$ signaling peptide (MALPVTALLLPLALLLHAARP) followed by a FLAG 352 tag (DYKDDDDK) and either an anti-CD19 (FMC63) (26), anti-ALPPL2 (M25, M25 ADLF, or 
$353 \mathrm{M} 25^{\mathrm{FYI}}$ ) (9), anti-MCAM (M1 or M40) (13), or anti-mesothelin (m912) (27) scFv. CARs were then

354 fused to either the CD8 $\alpha$ hinge/transmembrane domain, 4-1BB intracellular domain, and CD3 $\zeta$

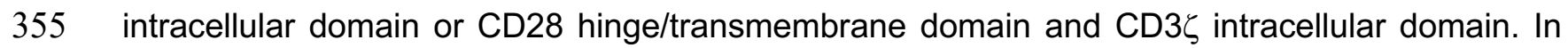

356 order to determine expression, a T2A self-cleaving peptide preceding eGFP was attached to CAR

357 coding sequence. In some experiments, CARs with a c-Myc-tag (EQKLISEEDL) instead of the

358 FLAG tag and a GS linker tethered eGFP instead of T2A-eGFP were used. All constitutive CARs

359 were cloned into a modified pHR'SIN:CSW vector containing a SFFV promoter. The ALPPL2

360 synNotch was constructed as previously described (12) using the anti-ALPPL2 (M25 ${ }^{\mathrm{FYIA}}$ ) scFv.

361 The ALPPL2-synNotch was cloned into a modified pHR'SIN:CSW vector containing a PGK

362 promoter. Inducible CAR response elements were constructed by subcloning the CAR-T2A-eGFP

363 sequences into a modified pHR'SIN:CSW vector carrying five copies of the Gal4 DNA binding

364 domain target sequence (GGAGCACTGTCCTCCGAACG) 5' to a minimal CMV promoter. These

365 vectors also included a PGK promoter constitutively driving the expression of either of the

366 fluorescent reporters mCherry or BFP. For ectopic expression of ALPPL2, ALPI, and mesothelin,

367 coding sequences corresponding to NP_112603.2 (ALPPL2), NP_001622.2 (ALPI), and

368 NP_001170826.1 (mesothelin) were cloned into a modified pHR'SIN:CSW vector containing a

369 SFFV promoter. For ALPPL2 and ALPI, a FLAG tag was inserted $3^{\prime}$ of their predicted signaling

370 peptides (MQGPWVLLLLGLRLQLSLG for both). To establish non-perturbing nuclear fluorescent

371 labeling, Sv40 NLS (PKKKRKV) was fused 5' to mKate2 via a linker sequence (DPPVAT) and

372 cloned into a modified pHR'SIN:CSW vector containing a EF1 $\alpha$ promoter. To establish

373 doxycycline controlled expression of ALPPL2, FLAG tagged ALPPL2 was cloned into a modified

374 pHR'SIN:CSW vector containing a TRE3GS inducible promoter with constitutive downstream

375 cassette of a SFFV promoter driving the expression of rtTA3. To enable genetic knockout (KO) of

376 MCAM, the gRNA sequence GAGGCGCAGCTCCCGGGCTGG was cloned into the pL-

377 CRISPR.EFS.GFP vector, which was a gift from Benjamin Ebert (Addgene plasmid \#57818). AP- 
378 1, NFAT, and NFאB response elements and the minimal promoters used for $\mathrm{T}$ cell activity

379 reporters were a gift from Peter Steinberger (Addgene plasmid \#118031, 118094, and 118095)

380 and were cloned together with mCherry into a modified pHR'SIN:CSW vector. All constructs were

381 cloned via In-Fusion cloning (Takara Bio).

382

383 Lentiviral Production and Cell Lines

384 To produce pantropic VSV-G pseudotyped lentivirus, Lenti-X 293T cells (Takara Bio) were

385 transfected with a pHR'SIN:CSW transgene expression vector and the viral packaging plasmids

386 pCMVdR8.91 and pMD2.G using TransIT®-Lenti Transfection Reagent (Mirus Bio LLC).

387 Lentivirus containing medium was harvested $48 \mathrm{hrs}$ post-transfection. ALPPL2 ${ }^{+}, \mathrm{ALPI}^{+}, \mathrm{CD}^{+}{ }^{+}$, 388 and Mesothelin ${ }^{+}$K562 (ATCC) target cells lines were generated by lentiviral transduction followed 389 by fluorescence-activated cell sorting (FACS) based on cell surface protein expression. ALPPL2 ${ }^{+}$ 390 K562 cells were sorted based on FLAG tag expression. Generation of MCAM KO K562 target 391 cells was performed by lentiviral transduction with a vector carrying sgRNA-Cas9-P2A-eGFP 392 followed by FACS based on eGFP ${ }^{+}$CD146(MCAM)- cells. Human mesothelioma cell lines, M28 393 (epithelioid) and VAMT-1 (sarcomatoid), were originally obtained from Dr. Brenda Gerwin's lab at 394 the National Cancer Institute. Mesothelin ${ }^{+}$M28 target cells were generated by lentiviral 395 transduction followed by FACS based on cell surface mesothelin expression. To enable real-time 396 counting of M28 and VAMT-1 target cells, these cell lines were tagged with a non-perturbing 397 fluorescent mKate2 reporter through lentiviral transduction followed FACS purification of mKate2+ 398 cells. Inducible ALPPL2 ${ }^{+}$K562 target cells were generated via lentiviral transduction followed by 399 single-cell sorting of doxycycline treated cells into 96-well U-bottom plates. Clonal lines were 400 assessed for dose dependent ALPPL2 expression, as defined by cell surface FLAG tag levels, 401 and the most uniform line was used for subsequent assays. T cell activation reporter lines for AP402 1, NFAT, and NF $\kappa B$ were generated through lentiviral transduction of Jurkat (clone E6-1) cells 
403 (ATCC) and sorted based on the low basal activity seen for these reporters. Lenti-X 293T cells

404 and M28 were cultured in DMEM (Gibco) with 10\% fetal bovine serum (MilliporeSigma), 40550 units $/ \mathrm{mL}$ Penicillin and $50 \mu \mathrm{g} / \mathrm{ml}$ Streptomycin (MP biochemicals), and 1X Sodium Pyruvate 406 (MilliporeSigma). K562s were cultured in IMDM (Gibco) with 10\% FBS, 50 units/mL Penicillin and $40750 \mu \mathrm{g} / \mathrm{ml}$ Streptomycin. Jurkat, M28, and VAMT-1 were cultured in RPMI-1640 (Gibco) with 10\% 408 FBS, 100 units $/ \mathrm{mL}$ Penicillin and $100 \mu \mathrm{g} / \mathrm{ml}$ Streptomycin, 1 X Glutamax (Gibco).

410 Isolation and Engineering of Human T cells

411 Primary $\mathrm{CD}^{+}$and $\mathrm{CD} 8^{+} \mathrm{T}$ cells were isolated from Fresh Human Peripheral Blood Leukapheresis 412 Packs (STEMCELL Technologies) using EasySep ${ }^{\mathrm{TM}}$ isolation kits (STEMCELL Technologies). T 413 cells were cryopreserved in RPMI-1640 with 20\% human AB serum (Valley Biomedical) and 10\% 414 DMSO (Fisher Scientific). After thawing, T cells were cultured in human T cell medium consisting 415 of X-VIVO 15 (Lonza), 5\% Human AB serum, and $10 \mathrm{mM}$ neutralized N-acetyl L-Cysteine 416 (MilliporeSigma) supplemented with 30 units/mL recombinant human IL-2 (R\&D Systems).

417 Primary human $\mathrm{CD}^{+}$or $\mathrm{CD}^{+} \mathrm{T}$ cells were thawed and left to recover for $24 \mathrm{hrs}$ after which they 418 were stimulated with Human T-Activator CD3/CD28 Dynabeads (Thermo Fisher Scientific) at a 419 1:1 cell:bead ratio. After an additional $24 \mathrm{hrs}$, the primary T cells were exposed to lentivirus for 24 420 hrs. At day 5 after T cell stimulation, the Dynabeads were removed and T cells were sorted to 421 carry to correct transgene composition. T cells were expanded and rested until day 10 for in vivo 422 experiments and day 14 for in vitro assays.

\section{Doxycycline inducible ALPPL2}

425 A clonal line of K562 cells carrying a doxycycline inducible ALPPL2 cassette was treated with 426 doxycycline (Abcam) at doses ranging between $0.1-10 \mathrm{ng} / \mathrm{mL}$ for 3 days. Surface expression 427 levels were assessed by flow cytometry through the FLAG-tag on ALPPL2 before being used in 428 T cell stimulation assays. 


\section{Activation, Proliferation, and Intracellular Cytokine Assays}

431 For in vitro stimulation assays of engineered T cells, effector cells were combined with targets

432 cells at a 1:1 ratio in 96-well U-bottom plates and centrifuged for $1 \mathrm{~min}$ at $400 \times \mathrm{g}$ to force

433 interaction of the cells. To assess T cell activation, cells were analyzed for surface expression of

434 CD69 16-24 hrs post target challenge. For proliferation assays, engineered T cells were stained

435 with CellTrace Violet (CTV) Cell Proliferation Kit (Thermo Fisher Scientific) prior to being

436 combined with target cells. CTV dilution was assessed 4 days post target challenge. Intracellular

437 cytokine (IC) assays using constitutive CARs, were set up as above but in the presence of $1 \mathrm{X}$

438 Brefeldin A solution (Thermo Fisher Scientific) and 1X Monensin solution (Biolegend). IC assays

439 using inducible CAR circuits were performed by incubating effector cells and targets for 16 hrs

440 before the addition of $1 \mathrm{X}$ Brefeldin A solution and 1X Monensin solution, in order to enable robust

441 CAR expression and trafficking to the cell surface before blocking protein transport. All

442 experiments were performed in T cell medium supplemented with 30 units/mL IL-2.

\section{Incucyte Killing Assay}

445 For in vitro engineered T cell killing assays, M28 or VAMT-1 expressing nuclear mKate2 were 446 seeded in 96-well flat-bottom plates. After $24 \mathrm{hrs}$ engineered T cells were added at an expected 447 effector:target ratio of 2:1. Plates were imaged every 2 hrs using the IncuCyte® S3 Live-Cell 448 Analysis System (Essen Bioscience) for a duration of 5 days. Three images per well at 10X 449 magnification were collected and analyzed using the IncuCyte $\circledR$ S3 Software (Essen Bioscience) 450 to detect and count the number of $m$ Kate $2^{+}$nuclei per image. Experiments were performed in 451 RPMI-1640 with 10\% FBS, 100 units/mL Penicillin and $100 \mu \mathrm{g} / \mathrm{ml}$ Streptomycin, 1 X Glutamax 452 supplemented with 30 units/mL IL-2. 
456 For scFv binding analysis, M28 and VAMT-1 mesothelioma cells in exponential growth phase 457 were incubated with M1 (MCAM) or M25 ${ }^{\text {FYIA }}$ (ALPPL2) scFv for 1h at RT, washed three times with 458 PBS, further incubated with a secondary anti-6xHis (4E3D10H2/E3, MA1-135-A647, Thermo 459 Fischer Scientific) at 1:1000 dilution for $1 \mathrm{~h}$ at RT, and washed three times with PBS before 460 analysis. For immunophenotyping, samples were stained for either $20-30 \mathrm{~min}$ at $4^{\circ} \mathrm{C}, 30 \mathrm{~min}$ at RT, 461 or $15 \mathrm{~min}$ at $37^{\circ} \mathrm{C}$ followed by $15 \mathrm{~min}$ at $\mathrm{RT}$, depending on the panel. For extracellular stains, cell 462 washes and final resuspension was performed with PBS supplemented with $2 \%$ FBS. IC stains 463 were performed using an Intracellular Fixation \& Permeabilization Buffer Set (Thermo Fisher 464 Scientific) per manufacturer's instructions. Dead cells were excluded with Draq7 (Abcam) or 465 Zombie NIR ${ }^{\mathrm{TM}}$ Fixable Viability Kit (Biolegend).

For immunophenotyping, the following antibodies were used: anti-CD146 (541467 10B2, 130-097-942, Miltenyi), anti-CD19 (HIB19,11-0199-41, eBioscience), anti-CD197 468 (G043H7, 353226, Biolegend), anti-CD223 (11C3C65, 369322, Biolegend), anti-CD27 (M-T271, 356424, Biolegend), anti-CD279 (EH12.2H7, 329906 and 329908, Biolegend), anti-CD3 (UCHT1, 300463, Biolegend), anti-CD366 (7D3, 563422, BD biosciences), anti-CD366 (F38-2E2, 345026,

471 Biolegend), anti-CD39 (A1, 328228, Biolegend), anti-CD4 (SK3, 563875, BD biosciences), anti472 CD45 (2D1, 368515, Biolegend), anti-CD45RA (HI100, 304150, Biolegend), anti-CD45RO 473 (UCHL1, 564291, BD biosciences), anti-CD62L (DREG-56, 304830 and 304822, Biolegend), anti474 CD69 (FN50, 564364, BD biosciences), anti-CD69 (FN50, 310910, Biolegend), anti-CD8a (RPA475 T8, 563796 and 563823, BD biosciences), anti-CD8a (SK1, 344706, Biolegend), anti-CD8a 476 (OKT8, 17-0086-42, eBioscience), anti-CD95 (DX2, 305644, Biolegend), anti-FLAG 477 (DYKDDDDK) tag (L5, 637310, Biolegend), anti-IFN- $\gamma$ (4S.B3, 563731, BD biosciences), anti-IL4782 (MQ1-17H12, 560707, BD biosciences), anti-Mesothelin (REA1057, 130-118-096, Miltenyi), 479 anti-Myc-tag (9B11, 2233S, Cell Signaling Technology), and anti-TNF (MAb11, 563996, BD 480 biosciences). Cells were analyzed using either Accuri ${ }^{\mathrm{TM}} \mathrm{C6}$, LSR II SORP, FACSymphony X50 
481 SORP or, for cell sorting, FACSAria II SORP, FACSAria IIlu SORP, or FACSAria Fusion SORP

482 (all BD Biosciences). Cell counts were performed using flow cytometry with CountBright Absolute

483 Counting Beads (Thermo Fisher Scientific) per manufacturer's instructions. Data was analyzed

484 using FlowJo software (BD Biosciences).

485

\section{Xenograft Tumor Models}

487 NOD.Cg-Prkdc $c^{\text {scid }} / / 2 \mathrm{rg}^{\text {tmiWjil} / S z J}$ (NSG) mice were implanted with $4 \times 10^{6} \mathrm{M} 28$ tumor cells 488 subcutaneously of the right flank. Seven days after tumor implantation, $3 \times 10^{6}$ engineered primary 489 human $\mathrm{CD}^{+}$and $\mathrm{CD} 8^{+} \mathrm{T}$ cells (total of $6 \times 10^{6} \mathrm{~T}$ cells) were infused i.v. through tail vein injection. 490 Tumor size was monitored via caliper weekly and tumor weight was measured at completion of 491 experiment. For immunophenotypic analysis, spleens were manually dissociated and subjected 492 to red blood cell lysis (ACK; KD medical). 
493 LIST OF SUPPLEMENTAL TABLES:

494

495 Supplemental Table S1. ALPPL2 expression in solid tumors.

496 Supplemental Table S2. Tissue specificity of ALPPL2 expression.

497 Supplemental Table S3. IHC study of MCAM expression in FFPE mesothelioma tissue arrays.

498 Supplemental Table S4. MCAM co-expression in ALPPL2 positive mesothelioma. 
499 Supplementary Table S1. ALPPL2 expression in solid tumors. IHC was performed on FFPE

500 tumor tissues using a mouse monoclonal antibody (LifeSpan clone SPM593, catalogue\# LS-

501 C390148-20). The number and percentage of ALPPL2 positive cases are indicated. For

502 reference, we also analyzed a public protein expression database, the Human Protein Atlas

503 (https://www.proteinatlas.org), and listed the result of ALPPL2 expression by IHC study using the

504 validated rabbit polyclonal antibody (MilliporeSigma HPA038764). NA: not available.

\begin{tabular}{|c|c|c|}
\hline Tumor Category & This study & Protein Atlas \\
\hline Mesothelioma & $39 / 91(42.86 \%)$ & N/A \\
\hline Ovarian cancer & $36 / 60(60 \%)$ & $7 / 12(58.33 \%)$ \\
\hline Pancreatic cancer & $18 / 50(36 \%)$ & $2 / 10(20 \%)$ \\
\hline Gastric cancer & $13 / 72(18.06 \%)$ & $3 / 11(27.27 \%)$ \\
\hline Seminoma & $11 / 11(100 \%)$ & $9 / 11(81.82 \%)$ \\
\hline
\end{tabular}

506 
507 Supplementary Table S2. Tissue specificity of ALPPL2 expression. The biotin-labeled M25 $5^{\mathrm{FYIA}}$

$508 \mathrm{scFv}$ and a biotin-labeled non-binding scFv (control) were used to stain normal human frozen

509 tissue arrays. Each tissue type has duplicated cores on the array. Staining results of the M25 FYIA

$510 \mathrm{scFv}$ were compared with that of the control scFv to determine positive or negative staining. N:

511 negative staining.

512

\begin{tabular}{|c|c|c|}
\hline Organ & Core 1 & Core 2 \\
\hline Salivary & $\mathrm{N}$ & $\mathrm{N}$ \\
\hline Esophagus & $\mathrm{N}$ & $\mathrm{N}$ \\
\hline Rectum & $\mathrm{N}$ & $\mathrm{N}$ \\
\hline Stomach & $\mathrm{N}$ & $\mathrm{N}$ \\
\hline Kidney & $\mathrm{N}$ & $\mathrm{N}$ \\
\hline Skeletal muscle & $\mathrm{N}$ & $\mathrm{N}$ \\
\hline Skin & $\mathrm{N}$ & $\mathrm{N}$ \\
\hline Testis & $\mathrm{N}$ & $\mathrm{N}$ \\
\hline Placenta & Positive & Positive \\
\hline Breast & $\mathrm{N}$ & $\mathrm{N}$ \\
\hline Cervix & $\mathrm{N}$ & $\mathrm{N}$ \\
\hline Uterus & $\mathrm{N}$ & $\mathrm{N}$ \\
\hline Spleen & $\mathrm{N}$ & $\mathrm{N}$ \\
\hline Lung & $\mathrm{N}$ & $\mathrm{N}$ \\
\hline Cerebellum & $\mathrm{N}$ & $\mathrm{N}$ \\
\hline Nerve & $\mathrm{N}$ & $\mathrm{N}$ \\
\hline Thyroid gland & $\mathrm{N}$ & $\mathrm{N}$ \\
\hline Pancreas & $\mathrm{N}$ & $\mathrm{N}$ \\
\hline Ovary & $\mathrm{N}$ & $\mathrm{N}$ \\
\hline Prostate & $\mathrm{N}$ & $\mathrm{N}$ \\
\hline
\end{tabular}


514 Supplementary Table S3. IHC study of MCAM expression in FFPE mesothelioma tissue arrays.

515 The number and percentage of each type of staining patterns are indicated. MCAM was detected

516 by both monoclonal (Abcam clone EPR3208, catalogue\# ab75769) and polyclonal antibody (pAb)

517 (Abcam, catalogue\# ab228487). The difference in percentage between mAb and pAb may reflect

518 difference in sensitivity of antigen/epitope detection in FFPE tissues.

519

\begin{tabular}{|c|c|c|}
\hline Staining Result & $\begin{array}{c}\text { Anti-MCAM } \\
\text { (Rabbit mAb) }\end{array}$ & $\begin{array}{c}\text { Anti-MCAM } \\
\text { (Rabbit pAb) }\end{array}$ \\
\hline Positive & $31(38.27 \%)$ & $73(75.26 \%)$ \\
\hline Negative & $50(61.73 \%)$ & $24(24.74 \%)$ \\
\hline Total tissue cores studied & 81 & 97 \\
\hline
\end{tabular}


521 Supplementary Table S4. MCAM co-expression in ALPPL2 positive mesothelioma. IHC study 522 using anti-ALPPL2 mouse mAb (LifeSpan clone SPM593) and anti-MCAM rabbit mAb (Abcam 523 clone EPR3208) or pAb (Abcam) was performed on FFPE tumor tissue arrays. Antibody pair 1:

524 Anti-ALPPL2 mouse mAb + Anti-MCAM rabbit mAb. Antibody pair 2: Anti-ALPPL2 mouse mAb + 525 Anti-MCAM rabbit pAb. The number and percentage of each type of staining patterns are 526 indicated.

527

\begin{tabular}{|c|c|c|}
\hline ALPPL2 positive/MCAM positive & $46(52.27 \%)$ & $58(80.56 \%)$ \\
\hline ALPPL2 positive/MCAM negative & $42(47.73 \%)$ & $14(19.44 \%)$ \\
\hline Total ALPPL2 positive tissue cores studied & 88 & 72 \\
\hline
\end{tabular}


Figure S1

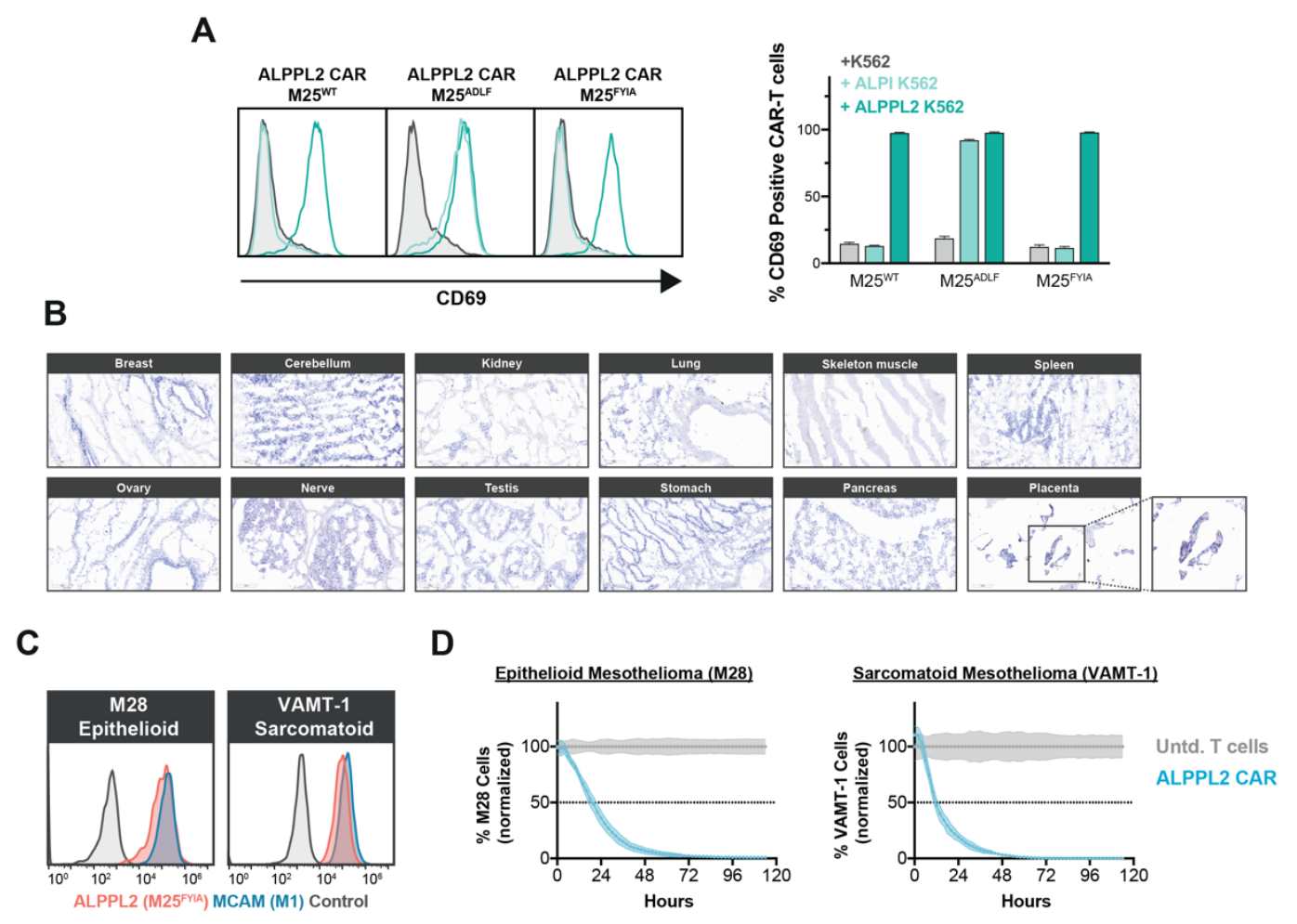

Supplementary Figure 1. ALPPL2 Tissue Expression and ALPPL2 CAR Specificity. (A)

533 Expression of the early activation marker CD69 in $\mathrm{CD} 8^{+} \mathrm{CAR}-\mathrm{T}$ cells expressing a BB $\zeta$ CAR with

534 either a M25 $5^{\mathrm{WT}}, \mathrm{M} 25^{\mathrm{ADLF}}$, or M25 $5^{\mathrm{FYIA}}$ scFv 24 hrs after stimulation with K562s expressing either

535 ALPI or ALPPL2. (B) IHC study of ALPPL2 expression in normal human frozen tissues using the $536 \mathrm{M} 25^{\mathrm{FYIA}}$ scFv. Binding was only observed in placental trophoblasts. Scale bar: $100 \mu \mathrm{m}$. (C) Cell

537 surface co-expression of ALPPL2 and MCAM in an epithelioid mesothelioma cell line M28 and a 538 sarcomatoid cell line VAMT-1 using M1 and M25 $5^{\mathrm{FIA}}$ scFvs. (D) Killing kinetics of epithelioid (M28) 539 and sarcomatoid (VAMT-1) mesothelioma tumor cells by CD8 ${ }^{+} \mathrm{T}$ cells expressing an ALPPL2 $540 \quad \operatorname{CAR}\left(\mathrm{M} 25^{\mathrm{FYIA}}\right)$ 


\section{Figure S2}

A

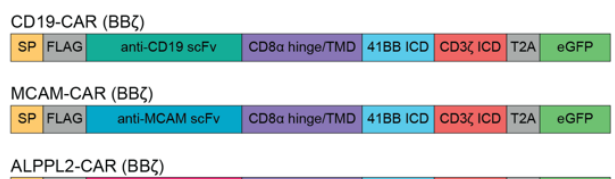

ALPPL2-CAR (BBל)

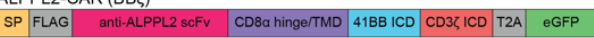

MESO-CAR (BBל)

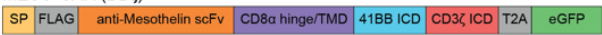

MESO-CAR (28Z)

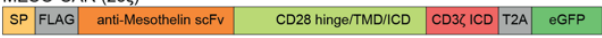

B
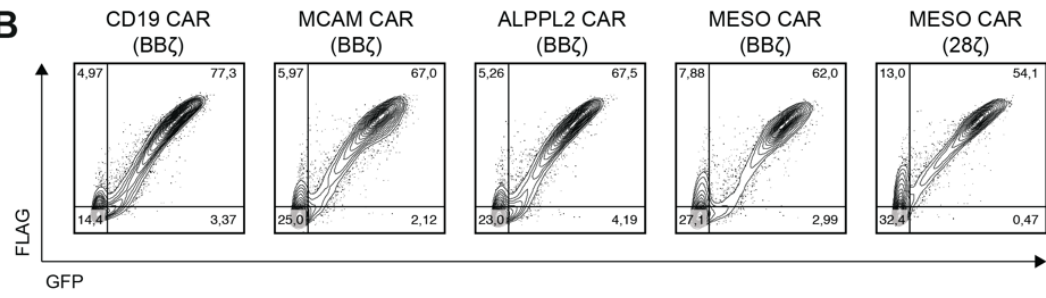

C

I cell Activation at $24 \mathrm{hrs}$.

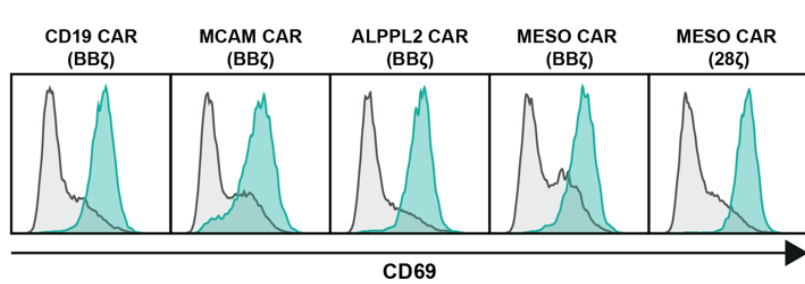

D $\underline{\text { I cell Proliferation at } 96 \mathrm{hrs} .}$

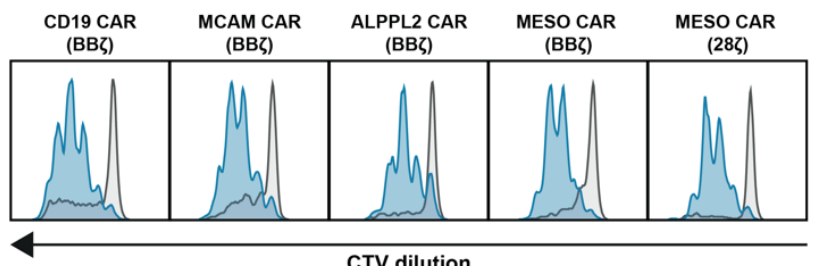

E

I cell Cytokine Production at $24 \mathrm{hrs}$.

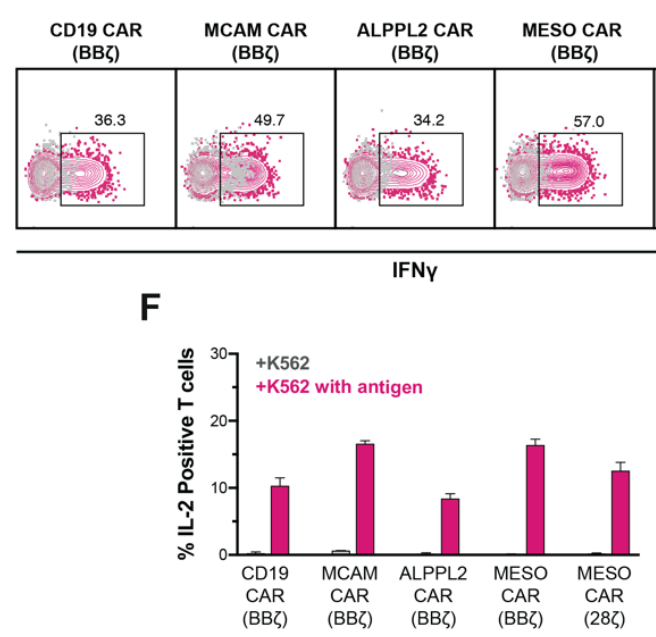

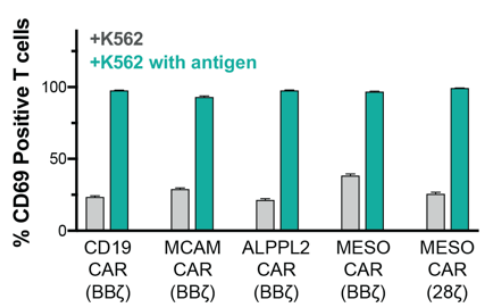

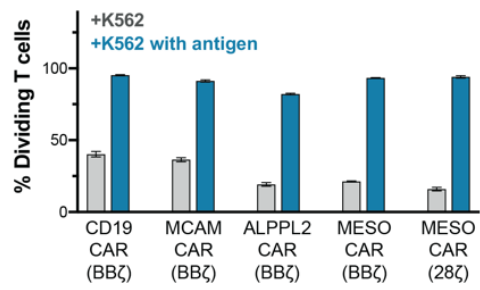

$80-$

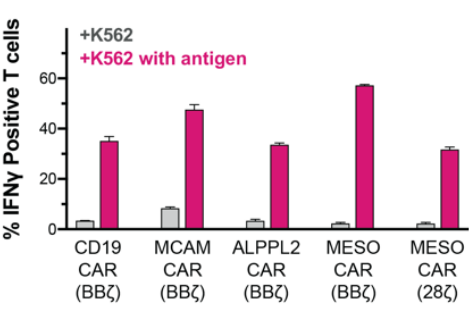

G

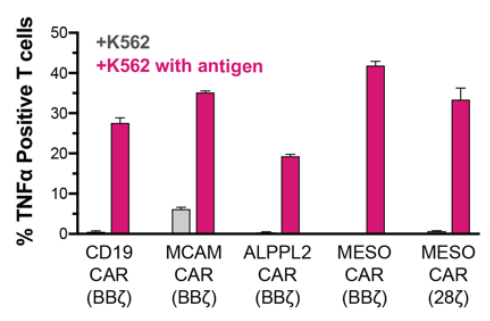


542 Supplementary Figure 2. Functional Characterization of Novel CARs (A) Design of CD19,

543 MCAM, ALPPL2, and Mesothelin CARs included in the study and (B) their expression in CD8 ${ }^{+} \mathrm{T}$

544 cells. $\mathrm{SP}=$ signaling peptide; FLAG = FLAG tag; ICD = intracellular domain; $\mathrm{TMD}=$

545 transmembrane domain. (C) Expression of the early activation marker CD69 in CD8 ${ }^{+}$CAR-T cells

$54624 \mathrm{hrs}$ after stimulation with K562s expressing the targeted antigen. (D) Proliferation of cell trace

547 violet (CTV) labelled CD8 ${ }^{+}$CAR-T cells four days after stimulation K562s expressing the cognate

548 antigen. (E) IFN $\gamma(\mathbf{F})$ IL-2 and (G) TNF $\alpha$ production in CD8 ${ }^{+}$CAR-T cells 24 hrs after stimulation

549 with K562s expressing the cognate antigen. 


\section{Figure S3}
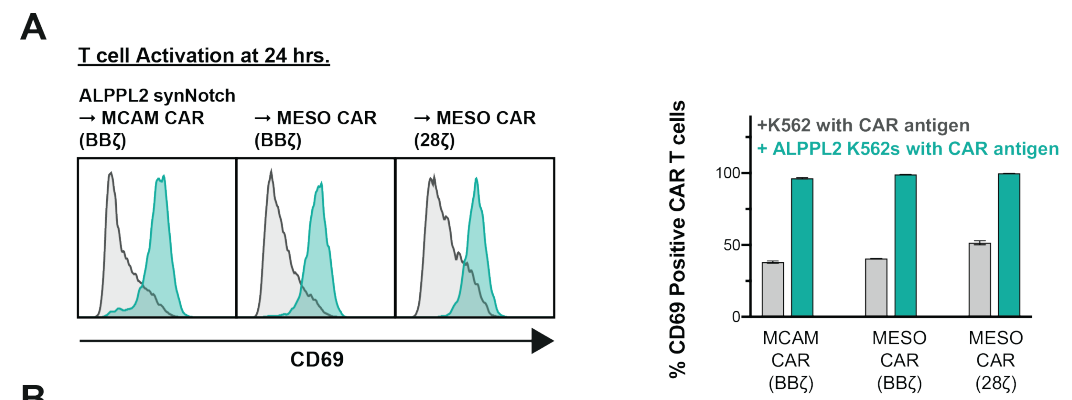

B
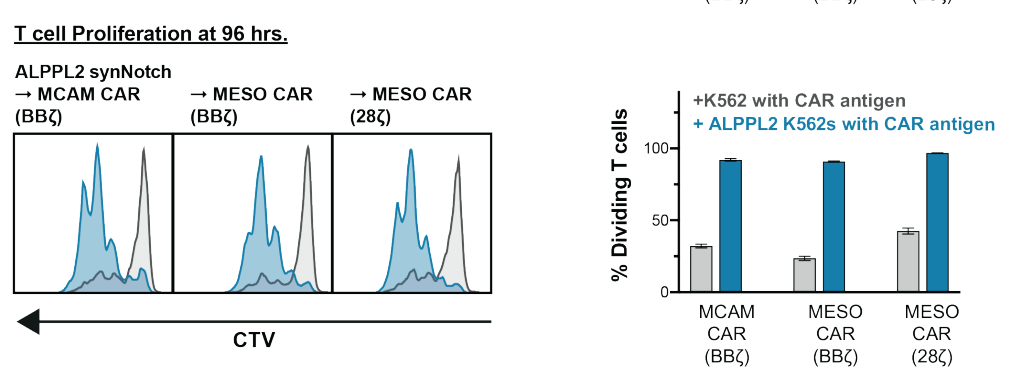

C
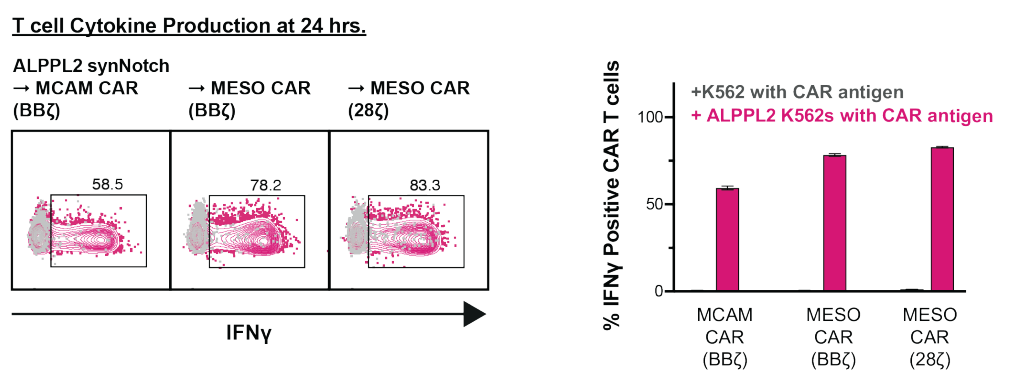

D
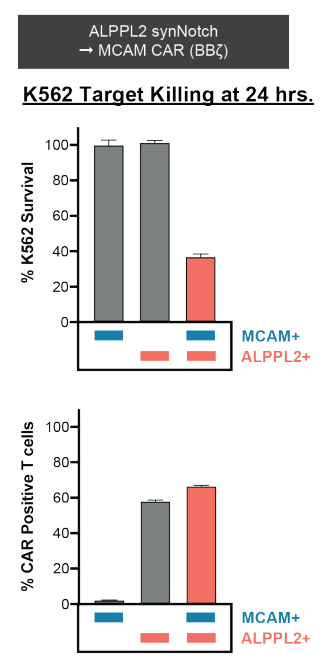

E
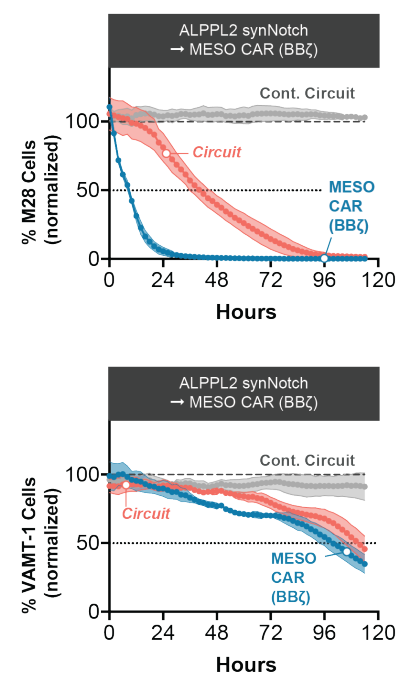

$\mathbf{F}$
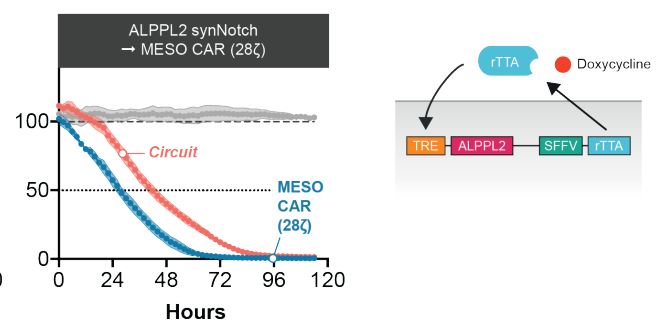

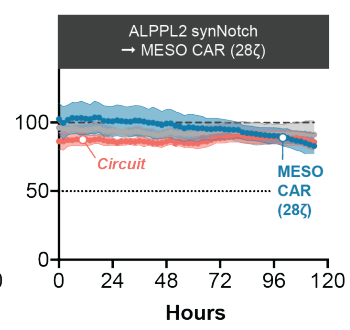

551 Supplementary Figure 3. Functional Characterization of ALPPL2-synNotch CAR circuits.

552 (A) $\mathrm{CD}^{+} \mathrm{T}$ cells were engineered to express an ALPPL2 synNotch together with a genetic circuit 
553 encoding for either a $\mathrm{MCAM}(\mathrm{BB} \zeta)$ or mesothelin $(\mathrm{BB} \zeta$ or $28 \zeta)$ CAR analyzed for CD69 expression

554 in CAR positive cells after 24 hrs of stimulation with K562s expressing the cognate CAR antigen

555 +/- ALPPL2. (B) Proliferation of CTV labelled CD8 ${ }^{+}$T cells ALPPL2 synNotch CAR circuits four

556 days after stimulation with K562s expressing the cognate CAR antigen +/- ALPPL2. (C) IFN $\gamma$

557 production in $\mathrm{CD}^{+} \mathrm{T}$ cells ALPPL2-synNotch CAR circuits four after stimulation with K562s

558 expressing the cognate CAR antigen +/- ALPPL2. (D) Target killing of K562s expressing MCAM,

559 ALPPL2, or the combination of the two by $C D 8^{+}$T cells expressing an ALPPL2 synNotch MCAM

560 CAR $(\mathrm{BB} \zeta)$ circuit after $24 \mathrm{hrs}$ of co-culture. Percentage CAR positive T cells was determined by

561 GFP expression. (E) Incucyte assay showing killing kinetics of epithelioid (M28) and sarcomatoid

562 (VAMT-1) mesothelioma tumor cells by $\mathrm{CD}^{+} \mathrm{T}$ cells expressing a $\mathrm{BB} \zeta$ mesothelin- or $28 \zeta$

563 mesothelin CARs constitutively or through an. (F) Genetic circuit for doxycycline inducible

564 ALPPL2 surface expression. 
Figure S4

A

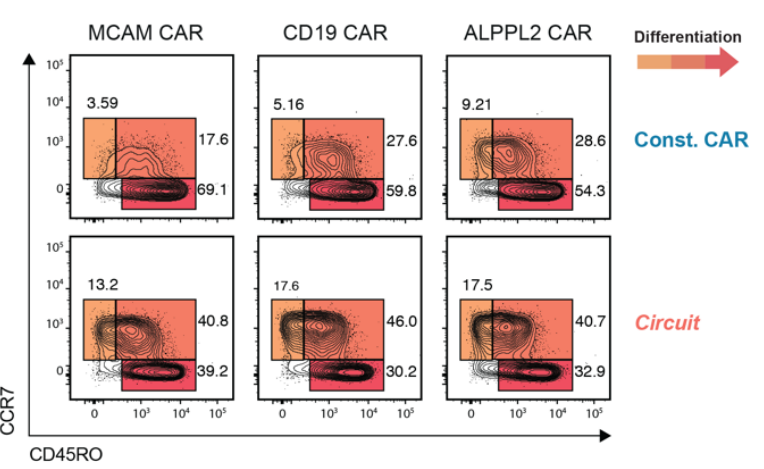

C

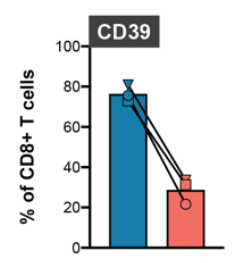

E
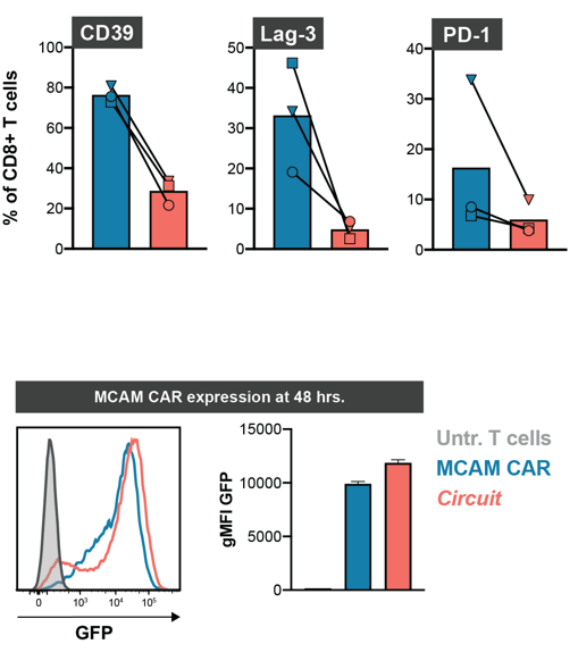

F
B

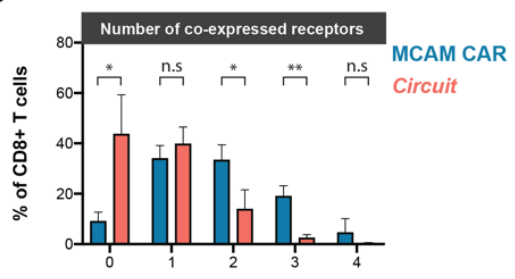

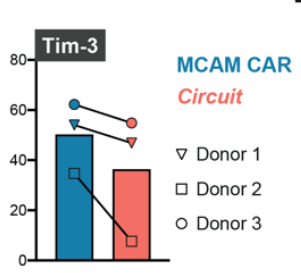

D
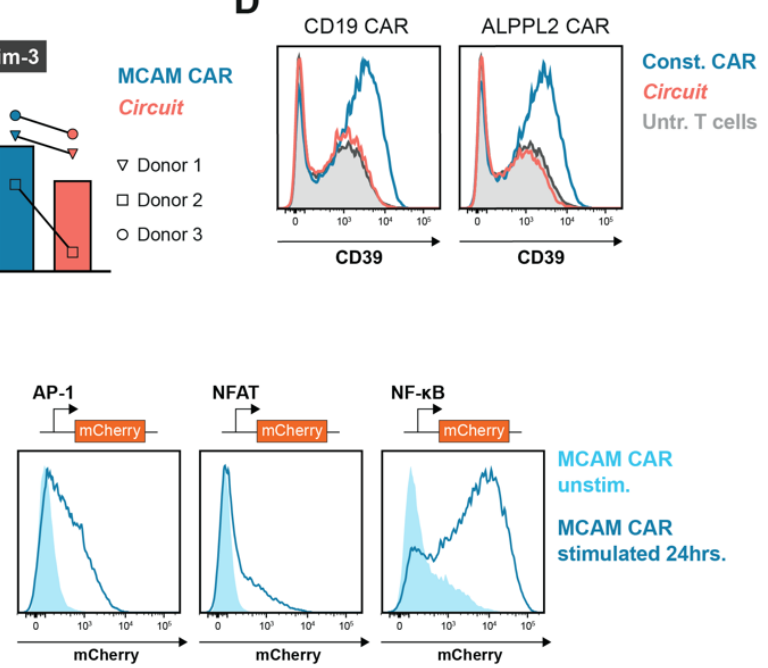

Supplementary Figure 4. Phenotypic Analysis of CAR and SynNotch CAR T cells Prior to

Antigen Exposure. (A) Composition of $\mathrm{T}_{\mathrm{SCM}}, \mathrm{T}_{\mathrm{CM}}$, and $\mathrm{T}_{\mathrm{EM}}$ in non-antigen exposed $\mathrm{CD} 8^{+} \mathrm{T}_{\text {cells }}$ engineered to express a MCAM, CD19, or ALPPL2 CAR either constitutively or through ALPPL2 synNotch circuit 14 days post initial CD3/CD28 Dynabead stimulation. Data linked to figure Fig. 2C. (B) Co-expressional pattern and (C) fraction of cells positive for CD39, Lag-3, PD-1, Tim-3 in

571 non-antigen exposed $\mathrm{CD}^{+} \mathrm{T}$ cells from three different donors engineered to express a MCAM

572 CAR either constitutively or through ALPPL2 synNotch circuit 14 days post initial CD3/CD28

573 Dynabead stimulation. Data linked to Fig. 2D. (D) Expression of CD39 in CD8+ T cells engineered

574 to express a CD19-, ALPPL2 CAR either constitutively or through ALPPL2 synNotch circuit 14 575 days post initial CD3/CD28 Dynabead stimulation. (E) MCAM CAR expression levels in CD8 ${ }^{+} \mathrm{T}$ 
576 cells stimulated with K562s expressing both MCAM and ALPPL2 for 48 hrs as determined by GFP

577 tethered to the CAR. (F) AP-1, NFAT, or NF-kB response element activity in Jurkat cells

578 constitutively expressing a MCAM-CAR 24 hrs after stimulation with MCAM positive K562s.

579 Statistics were calculated using unpaired (B) or paired (C) Student's t-test. Data is shown as

580 mean $\pm S D$. ${ }^{*} \mathrm{P} \leq 0.05 ;{ }^{* *} \mathrm{P} \leq 0.01$; n.s.; not significant. 


\section{Figure S5}

A

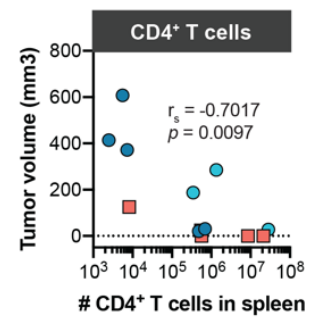

\section{CD8 ${ }^{+} \mathrm{T}$ cells}

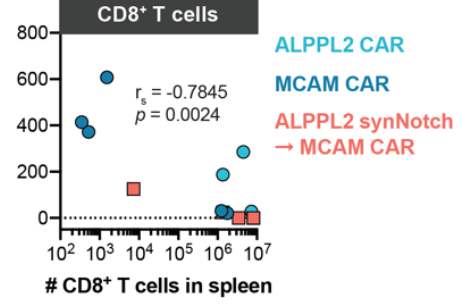

B

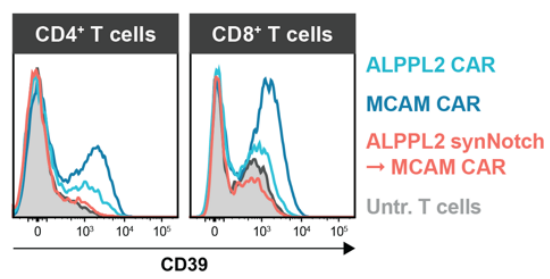

582 Supplementary Figure 5. Persistence of CAR T cells and SynNotch CAR Circuit T cells in

583 Treated Tumor-bearing Mice. (A) Correlation between final tumor volume and number of human

$584 \mathrm{CD}^{+}$or $\mathrm{CD}^{+} \mathrm{T}$ cells in the spleen at take down. Correlation was determined using Spearman's

585 rank correlation coefficient. (B) CD39 expression in CD4 ${ }^{+}$and $\mathrm{CD}^{+} \mathrm{T}$ cells engineered with an

586 ALPPL2 CAR, MCAM CAR, or ALPPL2 synNotch regulating MCAM CAR expression 10 days

587 post initial CD3/CD28 Dynabead stimulation. 


\section{References}

590 1. E. Jacoby, S. A. Shahani, N. N. Shah, Updates on CAR T-cell therapy in B-cell 591 malignancies. Immunol. Rev. 290, 39-59 (2019).

592 2. M. Carbone et al., Mesothelioma: Scientific clues for prevention, diagnosis, and therapy. 593 CA Cancer J Clin. 69, 402-429 (2019).

594 3. F. Nicolini et al., Malignant Pleural Mesothelioma: State-of-the-Art on Current Therapies 595 and Promises for the Future. Front Oncol. 9, 1519 (2019).

596 4. C. J. de Gooijer, F. J. Borm, A. Scherpereel, P. Baas, Immunotherapy in Malignant Pleural 597 Mesothelioma. Front Oncol. 10, 187 (2020).

598 5. P. S. Adusumilli et al., Regional delivery of mesothelin-targeted CAR T cell therapy generates potent and long-lasting CD4-dependent tumor immunity. Sci Transl Med. 6, 261ra151-261ra151 (2014).

6. P. C. Schuberth et al., Treatment of malignant pleural mesothelioma by fibroblast activation protein-specific re-directed T cells. J Transl Med. 11, 187 (2013).

7. R. R. Meyerhoff et al., Impact of mesothelioma histologic subtype on outcomes in the Surveillance, Epidemiology, and End Results database. J. Surg. Res. 196, 23-32 (2015).

8. F. An et al., Targeted drug delivery to mesothelioma cells using functionally selected internalizing human single-chain antibodies. Mol. Cancer Ther. 7, 569-578 (2008).

9. Y. Su et al., ALPPL2 is a highly specific and targetable tumor cell surface antigen. bioRxiv,

609 10. Y. Bi et al., Identification of ALPPL2 as a Naive Pluripotent State-Specific Surface Protein Essential for Human Naive Pluripotency Regulation. Cell Rep. 30, 3917-3931.e5 (2020).

11. A. Hyrenius-Wittsten, K. T. Roybal, Paving New Roads for CARs. Trends Cancer. 5, 583592 (2019).

613 12. K. T. Roybal et al., Precision Tumor Recognition by T Cells With Combinatorial Antigen$614 \quad$ Sensing Circuits. Cell. 164, 770-779 (2016). 
615 13. S. Bidlingmaier et al., Identification of MCAM/CD146 as the target antigen of a human 616 monoclonal antibody that recognizes both epithelioid and sarcomatoid types of 617 mesothelioma. Cancer Res. 69, 1570-1577 (2009).

618 14. I. M. Shih, M. Nesbit, M. Herlyn, R. J. Kurman, A new Mel-CAM (CD146)-specific 619 monoclonal antibody, MN-4, on paraffin-embedded tissue. Mod. Pathol. 11, 1098-1106 620 (1998).

621 15. X. Yan et al., A novel anti-CD146 monoclonal antibody, AA98, inhibits angiogenesis and 622 tumor growth. Blood. 102, 184-191 (2003).

623 16. R. A. Metcalf et al., p53 and Kirsten-ras mutations in human mesothelioma cell lines. Cancer Res. 52, 2610-2615 (1992).

625 17. J. Lv, P. Li, Mesothelin as a biomarker for targeted therapy. Biomark Res. 7, 18 (2019).

626 18. T. Eguchi et al., Cancer antigen profiling for malignant pleural mesothelioma immunotherapy: expression and coexpression of mesothelin, cancer antigen 125, and Wilms tumor 1. Oncotarget. 8, 77872-77882 (2017).

19. S. Inaguma et al., Comprehensive immunohistochemical study of mesothelin (MSLN) using different monoclonal antibodies 5B2 and $\mathrm{MN}-1$ in 1562 tumors with evaluation of its prognostic value in malignant pleural mesothelioma. Oncotarget. 8, 26744-26754 (2017).

632 20. A. Ajina, J. Maher, Strategies to Address Chimeric Antigen Receptor Tonic Signaling. Mol. Cancer Ther. 17, 1795-1815 (2018).

634 21. J. Eyquem et al., Targeting a CAR to the TRAC locus with CRISPR/Cas9 enhances tumour rejection. Nature. 543, 113-117 (2017).

636 22. A. H. Long et al., 4-1BB costimulation ameliorates $\mathrm{T}$ cell exhaustion induced by tonic signaling of chimeric antigen receptors. Nat. Med. 21, 581-590 (2015).

638 23. D. Gomes-Silva et al., Tonic 4-1BB Costimulation in Chimeric Antigen Receptors Impedes T Cell Survival and Is Vector-Dependent. Cell Rep. 21, 17-26 (2017). 
640 24. W. Ruan, A. Sassoon, F. An, J. P. Simko, B. Liu, Identification of clinically significant tumor 641 antigens by selecting phage antibody library on tumor cells in situ using laser capture 642 microdissection. Mol. Cell Proteomics. 5, 2364-2373 (2006).

643 25. F. An et al., Targeted drug delivery to mesothelioma cells using functionally selected 644 internalizing human single-chain antibodies. Mol. Cancer Ther. 7, 569-578 (2008).

645 26. I. C. Nicholson et al., Construction and characterisation of a functional CD19 specific single 646 chain Fv fragment for immunotherapy of B lineage leukaemia and lymphoma. Mol. Immunol.

$647 \quad 34,1157-1165(1997)$.

648 27. Y. Feng et al., A novel human monoclonal antibody that binds with high affinity to mesothelin649 expressing cells and kills them by antibody-dependent cell-mediated cytotoxicity. Mol. $650 \quad$ Cancer Ther. 8, 1113-1118 (2009). 